Tôhoku Math. Journ.

26 (1974), 95-131.

\title{
SUR LES ל-FONCTION D'EPSTEIN POUR DES OPÉRATEURS ELLIPTIQUES DÉGÉNÉRÉS
}

\author{
NoRIO SHIMAKURA
}

(Received March 22, 1973)

Introduction. Soit $A$ un opérateur différentiel du second ordre défini dans un ouvert borné $\Omega$ de $R^{n}(n \geqq 2)$ à frontière régulière $S$. Supposons que $A$ soit fortement elliptique dans $\Omega$ mais dégénéré partout sur $S$ en toutes les directions. Si $A$ est réalisable comme un opérateur auto-adjoint strictement positif dans $L^{2}(\Omega)$, il est intéressant de rechercher son spectre. Sous une hypothèse convenable sur la dégénéréssance (par exemple l'Hypothèse III dans le $\$ 1$ ), $A^{-1}$ devient compact et donc le spectre de $A$ est une suite des valeurs propres dont chacune est de multiplicité finie:

$$
0<\lambda_{1} \leqq \lambda_{2} \leqq \cdots \leqq \lambda_{j} \leqq \cdots \uparrow \infty \text {. }
$$

Posons

$$
N(\lambda)=\sum_{\lambda_{j} \leqq \lambda} 1, \quad \text { pour } \lambda>0 .
$$

Alors, le comportement asymptotique de $\lambda_{j}$ lorsque $j \rightarrow \infty$ se reflète dans celui de $N(\lambda)$ lorsque $\lambda \rightarrow \infty$ et vice versa.

Baouendi et Goulaouic [1] donnèrent un critère pour que

$$
N(\lambda) \sim \text { Cte. } \lambda^{n / 2}, \text { lorsque } \lambda \rightarrow \infty,
$$

c'est-à-dire, pour que $N(\lambda)$ croisse analoguement au cas où $A$ ne soit pas dégénéré. Pour fixer les idées, soit $A$ de la forme

$$
(A u)(x)=-\sum_{j, k=1}^{n} \frac{\partial}{\partial x_{j}}\left(a^{j k}(x) \frac{\partial u}{\partial x_{k}}\right)+c(x) u(x) .
$$

Alors, la formule asymptotique (0.3) est vraie si et seulement si

$$
\int_{\Omega}\left(\operatorname{dét}\left(a^{j k}(x)\right)\right)^{-1 / 2} d x<\infty .
$$

Dans les notations de ce mémoire, ce critère est équivalente à l'intégrabilité de $\phi(x)^{-n / 2} \operatorname{sur} \Omega$, et celle-ci est violée.

Le comportement exact de $N(\lambda)$ fut obtenu par Nordin [5]. Il a considéré la forme quadratique $(A u, u)_{L^{2}(\Omega)}$ pour les $u \in D(A)$, et l'a approximée, dans un voisinage de $S$, par des autres formes quadratiques plus concrètes. 
Et, il a réduit la question à la recherche de la distribution asymptotique des zéros des fonctions de Whittaker.

Dans ce mémoire, nous envisageons la propriété méromorphe de la $\zeta$-fonction d'Epstein

$$
Z(\alpha)=\sum_{j=1}^{\infty} \lambda_{j}^{-\alpha}
$$

sous les Hypothèses I, II et III' dans le $\S 1$. Cette fonction est liée avec la fonction de Green $G(t, P, Q)$ de l'opérateur parabolique $\partial / \partial t+A$. Il s'agit d'évaluer $G(t, P, P)$ lorsque $t \downarrow 0$ et $P$ s'approche de la frontière $S$. Ce qui est très différent du cas où $A$ est non dégénéré est la nature de cette $G(t, P, P)$. Les connaissances classiques sur les fonctions de Green du cas non dégénéré ne sont plus utiles quand on considère $G(t, P, P)$ pour $P$ voisin de $S$.

Pour concevoir intuitivement cette différence, nous comparons les solutions fondamentales des trois opérateurs simples:

$$
\frac{\partial}{\partial t}-\frac{\partial^{2}}{\partial x^{2}}, \frac{\partial}{\partial t}-\frac{\partial}{\partial x}\left(x \frac{\partial}{\partial x} \cdot\right) \text { et } \frac{\partial}{\partial t}-\frac{\partial}{\partial x}\left(x \frac{\partial}{\partial x} \cdot\right)+c^{2} x \cdot,
$$

où $x$ est la variable spatiale positive, et $c$ est une constante positive. La solution fondamentale du premier est $(4 \pi t)^{-1 / 2} \exp \left\{-(x-y)^{2} / 4 t\right\}$, tandis que celles du deuxième et $\mathrm{du}$ troisième sont

$$
\begin{gathered}
\frac{1}{t} I_{0}\left(\frac{2 \sqrt{x y}}{t}\right) \exp \left(-\frac{x+y}{t}\right) \\
\text { et } \frac{c}{\sinh (c t)} I_{0}\left(\frac{2 c \sqrt{x y}}{\sinh (c t)}\right) \exp (-c(x+y) \operatorname{coth}(c t))
\end{gathered}
$$

respectivement. Les deux dernières sont certainement très différentes de la première au voisinage de $(x, y)=(0,0)$.

Dans le $\S 1$, nous précisons les hypothèses sur $A$, citons les résultats de Baouendi-Goulaouic et de Nordin (Théorèmes 1.1 et 1.2) et énonçons le résultat sur $Z(\alpha)$ (Théorème 1. 3). Dans le $\S 2$, nous construisons la fonction de Green $G(t, P, Q)$, et démontrons le Théorème 1.3 dans le $\S 3$ en pleines utilisations des calculs aux $\S \S 6$ et 7 . Let $\S \S 4$ et 5 sont consacrés aux préparations du $\S 6$.

Le Théorème 1.3 est une réponse partielle à la conjecture que l'auteur proposa dans [8].

1. Hypothèses et résultat. Soit $\Omega$ un ouvert borné de $R^{n}(n \geqq 2)$ à frontière $S$ une hypersurface de classe $C^{\infty}$. Supposons que $\Omega$ soit situé à un seul côté de $\Omega$. Soit $A$ un opérateur différentiel du second ordre de la forme 


$$
(A u)(x)=\frac{-1}{\sqrt{g(x)}} \sum_{j, k=1}^{n} \frac{\partial}{\partial x_{j}}\left\{\phi(x) \sqrt{g(x)} g^{j k}(x) \frac{\partial u(x)}{\partial x_{k}}\right\}+c(x) u(x),
$$

où les coefficients $\phi(x), g^{j k}(x)$ et $c(x)$ satisfassent aux trois hypothèses suivantes:

HYPотнऐ̀SE I. La matrice $\left(g^{j k}(x)\right)_{j, k=1}^{n}$ est symétrique réelle et définie positive uniformément sur $\bar{\Omega}$, et $g(x)=\operatorname{dét}\left(g^{j k}(x)\right)^{-1}$.

НYротнऐेSE II. $c(x)>0$ sur $\bar{\Omega}$.

HYPoTHEेSE III. $\phi(x)$ est positive dans $\Omega$, nulle identiquement sur $S$ et telle que grad $\phi(x)$ ne s'annule jamais sur $S$.

Pour simplicité, nous supposons que tous les $\phi(x), g^{j k}(x)$ et $c(x)$ soient de classe $C^{\infty}(\bar{\Omega})$, bien que le Théorème 1.3 , notre résultat, reste vrai si $S$ et les coefficients sont de classe $C^{n+3}$ au moins.

Munnissons à $\bar{\Omega}$ de la structure riemannienne définie par la métrique

$$
d l^{2}=\sum_{j, k=1}^{n} g_{j k}(x) d x_{j} d x_{k}, \quad \text { où } \quad\left(g_{j k}(x)\right)=\left(g^{j k}(x)\right)^{-1},
$$

et désignons par $d v$ l'élément volumique $\sqrt{g(x)} d x_{1} \cdots d x_{n}$. L'espace $L^{2}(\Omega)$ est la totalité des $u(x)$ de carrée sommable sur $\Omega$ par rapport à $d v$. Ecrivons encore par $A$ l'extension de Friedrichs dans $L^{2}(\Omega)$ de $A$ restreint à $C^{\infty}(\bar{\Omega})$. Alors, $A$ est auto-adjoint défini positif dans $L^{2}(\Omega)$ du domaine

$$
D(A)=\left\{u(x) \in H^{1}(\Omega) ; \phi(x) u(x) \in H^{2}(\Omega)\right\}
$$

$\left(H^{k}(\Omega)\right.$ est l'espace de Sobolev d'ordre $\left.k\right)$.

Soit

$$
0<\lambda_{1} \leqq \lambda_{2} \leqq \cdots \leqq \lambda_{j} \leqq \cdots \uparrow \infty
$$

la suite de toutes les valeurs propres de $A$ dont chacune est comptée répétamment autant que sa multiplicité. Posons

$$
N(\lambda)=\sum_{\lambda_{j} \leq \lambda} 1, \text { pour } \lambda>0 .
$$

Cette fonction est appelée la fonction comptoire. Alors, un problème se pose comme suit: Quel est le règle de la croissance de la j-ième valeur propre $\lambda_{j}$ lorsque $j \rightarrow \infty$ ?, ou ce qui est équivalente, comment croît-elle la fonction comptoire $N(\lambda)$ lorsque $\lambda \rightarrow \infty$ ?

Baouendi-Goulaouic [1] proposèrent premièrement une réponse à cette question:

ThÉrème 1.1. (Baouendi-Goulaouic [1]). Nous avons

$$
\lim _{j \rightarrow \infty}\left(j^{-2 / n} \lambda_{j}\right)=0, \quad \text { et }
$$




$$
\lim _{j \rightarrow \infty}\left(j^{\varepsilon-1 /(n-1)} \lambda_{j}\right)=\infty, \text { quel que soit } \varepsilon>0 .
$$

Ce Théorème nous fait apprendre que la croissance des $\lambda_{j}$ est strictement plus lente que celle des valeurs propres des opérateurs du second ordre non dégénérés (par exemple, le laplacien sous la condition de Dirichlet) mais qu'elle n'est pas moins lente, grosso-modo, que l'ordre $j^{1 /(n-1)}$.

Ensuite, la réponse complète fut donnée par Nordin [5]:

THEORÈME 1.2. (Nordin [5]). Lorsque $\lambda \uparrow \infty, N(\lambda)$ se comporte comme suit:

$$
\begin{aligned}
& \text { Si } n=2, \quad N(\lambda) \approx d_{2} \lambda \log \lambda, \\
& \text { Si } n \geqq 3, \quad N(\lambda) \sim d_{n} \lambda^{n-1},
\end{aligned}
$$

où les constantes $d_{n}$ sont données par

$$
d_{n}= \begin{cases}c_{1} / 4 & , \text { pour } n=2, \\ (n-1) c_{n-1} \int_{0}^{\infty}\left[\frac{\xi+1}{2}\right] \xi^{-n} d \xi, & \text { pour } n \geqq 3,\end{cases}
$$

avec

$$
\begin{array}{r}
c_{n-1}=\frac{\omega_{n-1}}{(2 \pi)^{n-1}} \int_{S}\left(\frac{\partial \phi}{\partial \nu}\right)^{(1-n) / 2} d S \text { et } \omega_{n-1}=\pi^{(n-1) / 2} \Gamma\left(\frac{n+1}{2}\right)^{-1}, \\
\text { pour } n \geqq 2,
\end{array}
$$

$\partial / \partial \nu$ désigne la dérivée conormale à $S$ par rapport à la structure riemannienne ci-dessus, et $[z]$ est la partie entière de $z$.

Nordin obtint ce Théorème par le principe du min.-max. des valeurs propres dû à $\mathrm{H}$. Weyl et par de certaines connaissances sur les distributions des zéros des fonctions de Whittaker.

Dans ce mémoire, nous voulons établir un résultat analogue à ceci mais sur une propriété méromorphe de la $\zeta$-fonction d'Epstein

$$
Z(\alpha)=\int_{0}^{\infty} \lambda^{-\alpha} d N(\lambda)=\sum_{j=1}^{\infty} \lambda_{j}^{-\alpha}, \quad \alpha \in C .
$$

La série est convergente si $\operatorname{Re} \alpha>n-1$ grâce au Théorème 1.1. Et elle est liée avec la fonction de Green $G(t, P, Q)$ de l'opérateur parabolique $\partial / \partial t+A$ comme suit

$$
Z(\alpha)=\frac{1}{\Gamma(\alpha)} \int_{0}^{\infty} t^{\alpha-1} d t \int_{\Omega} G(t, P, P) d v_{P} .
$$

Notre résultat s'énonce comme le Théorème 1.3. ci-dessous, mais nous avons dû remplacer l'Hypothèse III par une autre beaucoup plus restreinte:

HYPOTHÈSE III'. $\phi(x)$ satisfait à l'Hypothèse III, et de plus 
(1.13) $\phi(x)=$ dis. $(x, S)$, si dis. $(x, S) \leqq \delta$, pour un $\delta>0$, où dis. $(x ; S)$ est la distance géodésique de $x$ à $S$ par rapport à la métrique dl.

REmarque. Au lieu de (1.13), nous pouvons considérer une situation où $\phi(x)$ n'est pas exactement égale à dis. $(x, S)$, mais elle est une fonction de la dis. $(x, S)$ seule et satisfaisant à l'Hypothèse III. Alors, le Théorème 1.3 qui suit reste variable avec le facteur $|S|$ convenablement modifié selon le Théorème 1.2. Ce qui est essentiel dans notre méthode est que, comme nous verrons dans le $\S 2$, la variable dis. $(x, S)$ et les variables tangentielles à $S$ sont presque séparées dans l'opérateur $A$.

Si (1.13) a lieu, nous avons dans (1.10), $\partial \phi /\left.\partial \nu\right|_{s}=1$, donc

$$
\int_{S}\left(\frac{\partial \phi}{\partial \nu}\right)^{(1-n) / 2} d s=|S|=\text { l'aire de } S .
$$

THÉORÈme 1.3. Sous les Hypothèses I, II et III', nous avons

(i) Si $n=2$, alors

$$
\begin{aligned}
Z(\alpha) & -\frac{|S|}{4 \pi}(\alpha-1)^{-2} \\
& -\frac{1}{4 \pi}\left\{(4 \log 2+\gamma)|S|+\text { Pf. } \int_{\Omega} \phi(P)^{-1} d v_{P}\right\}(\alpha-1)^{-1}
\end{aligned}
$$

est une fonction holomorphe dans $\operatorname{Re} \alpha>3 / 4$, où $\gamma$ est le nombre d'Euler et Pf. signifie la partie finie de l'intégrale au sens de L. Schwartz (voir [6]);

(ii) Si $n \geqq 3$, alors $Z(\alpha)-A_{n}(\alpha-n+1)^{-1}$ est une fonction holomorphe dans $\operatorname{Re} \alpha>n-(3 / 2)$, où

$$
A_{n}=2(n-1)(4 \pi)^{(1-n) / 2} \Gamma\left(\frac{n-1}{2}\right)^{-1}|S| \int_{0}^{\infty}\left[\frac{\xi+1}{2}\right] \xi^{-n} d \xi\left(=(n-1) d_{n}\right),
$$

où [z] est la partie entière de $z$.

Admettons ce Théorème pour le moment. Si $n \geqq 3$, on redémontre la partie correspondante (1.8) du Théorème 1.2 d'après le théorème tauberien d'Ikehara. Mais si $n=2$, l'auteur n'est pas certain si la partie (i) du Théorème 1.3 entraîne (1.7) du Théorème 1.2 ou non. Nous proposons alors une question:

QUESTION. Soit $f(t)$ une fonction non-négative et non-décroissante sur $t \geqq 0$. Supposons que

$$
\int_{0}^{\infty} e^{-\alpha t} d f(t)-(\alpha-1)^{-2}-C(\alpha-1)^{-1}
$$

soit une fonction holomorphe de $\alpha$ dans $\operatorname{Re} \alpha>1-\varepsilon$ avec un $\varepsilon>0$, où 
$C$ soit une constante. Alors, quelles restrictions en plus sur $f(t)$ sont-elles nécessaires pour en conclure que

$$
\lim _{t \rightarrow \infty}\left\{\frac{f(t)}{t e^{t}}\right\}=1 ?
$$

Revenons au Théorème 1.3. La démonstration se divise en quelques étapes, elle sera finie dans le $\S 3$. Dans la reste de ce paragraphe, nous terminons une moitié qui est relativement facile.

Lemme 1.1. On ajoute, si nécessaire, KI à $A$ avec un K grand. Alors,

$$
\frac{1}{\Gamma(\alpha)} \int_{1}^{\infty} t^{\alpha-1} d t \int_{\Omega} G(t, P, P) d v_{P}
$$

est une fonction entière de $\alpha$.

Preuve. La théorie générale du semi-groupe holomorphe nous dit que, si $t>0, e^{-t A}$ applique $L^{2}(\Omega)$ dans $D\left(A^{k}\right)$ pour tout $k=1,2, \cdots$ et

$$
\left\|e^{-t \boldsymbol{A}}\right\|_{L^{2}(\Omega) \rightarrow D\left(A^{k}\right)} \leqq \text { Cte. } t^{-k} e^{-c t} \text {, avec un } c>0 \text {. }
$$

Mais on sait que $D\left(A^{k}\right) \subset H^{k}(\Omega)$ avec l'injection continue (voir [1]). Prenons $k=k_{0}=[n / 2]+1$ par exemple. Alors, par le lemme de Sobolev,

$$
\left\|e^{-t A}\right\|_{L^{2}(\Omega) \rightarrow C^{0}(\bar{\Omega})} \leqq M t^{-k_{0}} e^{-c t} .
$$

Donc, $e^{-t A}=\left(e^{-t A / 2}\right)\left(e^{-t A / 2}\right) *$ a la représentation par noyau continu $G(t, P, Q)$ $\operatorname{sur} \bar{\Omega} \times \bar{\Omega}$, et

$$
|G(t, P, Q)| \leqq M^{\prime} t^{-2 k_{0}} e^{-c t} \text { quels que soient }(P, Q) \in \bar{\Omega} \times \bar{\Omega} .
$$

D'où le résultat.

C.Q.F.D.

Lemme 1.2. Soit $C$ un compact quelconque dans l'intérieur de $\Omega$. Alors,

$$
\frac{1}{\Gamma(\alpha)} \int_{0}^{1} t^{\alpha-1} d t \int_{C} G(t, P, P) d v_{P}
$$

est méromorphe dans $\alpha$-plan complexe tout entier. Le pôle le plus à droite est $\alpha=n / 2$, il est simple et le résidu est égal à

$$
\frac{1}{\Gamma(n / 2)} \int_{C}\{4 \pi \phi(P)\}^{-n / 2} d v_{P} .
$$

Preuve. $A$ n'est plus dégénéré sur $C$, mais $A$ est uniformément fortement elliptique sur C. La construction d'Eidelman ([2]) ou plutôt de Minakshisundaram-Pleijel ([4]) nous garantit l'existence d'une solution fondamentale $\bar{\gamma}(\mathrm{t}, P, Q)$ de $\partial / \partial t+A$ telle que 


$$
\bar{\gamma}(t, P, P)=(4 \pi t)^{-n / 2}\left\{\phi(P)^{-n / 2}+t a_{1}(P)+\cdots+t^{N} a_{N}(P)+O\left(t^{N+1}\right)\right\}
$$

uniformément sur $C$. Nous verrons d'autre part, dans le $\S 2$, que

$$
G(t, P, P)-\bar{\gamma}(t, P, P)=O\left(e^{-c^{\prime} / t}\right)
$$

avec une constante $c^{\prime}>0$ dépendante essentiellement à dis. $(C, S)$. D'où l'énoncé du Lemme.

Prenons $\delta$ dans l'Hypothèse III' et écrivons

$$
\Omega_{\delta}=\{P \in \bar{\Omega} ; \operatorname{dis.}(P, S)<\delta\} \text {, et } \Omega_{\delta}^{\prime}=\bar{\Omega} \backslash \Omega_{\delta} .
$$

Et prenons, par exemple, $\Omega_{\delta / 7}^{\prime}$ comme $C$ dans le Lemme 1.2. Et posons

$$
Z_{0}(\alpha)=\frac{1}{\Gamma(\alpha)} \int_{0}^{1} t^{\alpha-1} d t \int_{\Omega_{\delta / 7}} G(t, P, P) d v_{P} .
$$

Alors, nous avons déjà vu les faits suivants:

(1.22) Si $n \geqq 3, Z(\alpha)-Z_{0}(\alpha)$ est holomorphe dans $\operatorname{Re} \alpha>n-(3 / 2)$.

$$
\left\{\begin{array}{l}
\text { Si par contre } n=2, \text { alors } \\
Z(\alpha)-Z_{0}(\alpha)-\frac{1}{4 \pi(\alpha-1)} \int_{\sigma_{\delta / 7}^{\prime}} \frac{1}{\phi(P)} d v_{P} \\
\text { est holomorphe dans } \operatorname{Re} \alpha>1 / 2 .
\end{array}\right.
$$

Nous avons ainsi réduit la question au comportement de $G(t, P, P)$ dans $\Omega_{\delta / 7}$ La fonction $Z_{0}(\alpha)$ sera étudiée dans le $\S 3$.

2. Fonction de Green. Dans ce paragraphe, nous allons construire la fonction de Green $G(t, P, Q)$ pour l'opérateur parabolique $\partial / \partial t+A$ avec $A$ défini par (1.1). La construction est faite d'abord dans un voisinage de la frontière $S$, et ensuite dans $\Omega$ tout entier.

$\bar{\Omega}$ est munie de la structure riemannienne définie par la métrique $d l^{2}=$ $\sum_{j, k=1}^{n} g_{j k}(x) d x_{j} d x_{k}$. Notons

$$
\Omega_{\delta}=\{P \in \bar{\Omega} ; \text { dis. }(P, S)<\delta\} \text { et } \Omega_{\delta}^{\prime}=\Omega \mid \bar{\Omega}_{\delta}, \text { pour } \delta>0,
$$

où la distance est celle par rapport à $d l$. Nous bornons d'abord la considération à un voisinage d'un point sur $S$.

Soit $P_{0}$ un point quelconque fixe de $S$ et prenons un voisinage $V$ dans $S$ de $P_{0}$ où un système de coordonnées locales $s=\left(s_{1}, s_{2}, \cdots, s_{n-1}\right)$ à l'origine $P_{0}$ est défini. Soit d'autre part $P$ un point quelconque de $\Omega_{\gamma}$. Si $\delta$ est petit, il existe une et une seule ligne géodésique $L$ issue de $P$ qui pénètre $S$ à un point $\bar{P}$ à la direction conormale à $S$. Désignons, de nouveau,

$$
x=\operatorname{dis.}(P, S)=\operatorname{dis.}(P, \bar{P}), \text { donc } 0 \leqq x<\delta .
$$


La correspondance de $P \Omega_{\delta} \rightarrow \bar{P} \in S$ est unique (c'est une projection). Posons donc

$$
U=\left\{P \in \Omega_{\delta} ; \quad \bar{P} \in V\right\} .
$$

Pour $P \in U$, soient $s=\left(s_{1}, s_{2}, \cdots, s_{n-1}\right)$ les coordonnées de $\bar{P}$. Ainsi, chaque point $P \in U$ est identifié avec le point $(s, x)$ de $\overline{R_{+}^{n}}$ par un difféomorphisme, et l'on écrit $P=(s, x)$.

Nous allons construire une paramétrix de $\partial / \partial t+A$ dans un tel $U$. $A$ s'écrit par ce nouveau système comme suit:

$$
\begin{aligned}
(A u)(s, x)= & \frac{-1}{\sqrt{g(s, x)}} \frac{\partial}{\partial x}\left(x \sqrt{g(s, x)} \frac{\partial u}{\partial x}\right) \\
& -\frac{x}{\sqrt{g(s, x)}} \sum_{\alpha, \beta=1}^{n-1} \frac{\partial}{\partial s_{\alpha}}\left\{\sqrt{g(s, x)} g^{\alpha \beta}(s, x) \frac{\partial u}{\partial s_{\beta}}\right\}+c(s, x) u,
\end{aligned}
$$

parce que $\phi(s, x)=x$ dans $U$ si $\delta>0$ est petit grâce à l'Hypothèse III' $^{\prime}$ dans le paragraphe précédent.

Soit $G(t, P, Q)$ la fonction de Green cherchée, c'est-à-dire, pour les $u_{0}(P)$ suffisamment régulière, nous ayons

$$
\left(e^{-t A} u_{0}\right)(P)=\int_{\Omega} G(t, P, Q) u_{0}(Q) d v_{Q}, \quad t>0,
$$

où $d v_{Q}$ est l'élément volumique de $\bar{\Omega}$ par rapport à $d l$. Supposons que le support de $u_{0}$ soit contenu dans $U$, et regardons (2.5) seulement pour $P \in U$. En posant

$$
v(t, P)=g(P)^{1 / 4}\left(e^{-t 4} u_{0}\right)(P) \quad \text { et } \quad v_{0}(P)=g(P)^{1 / 4} u_{0}(P),
$$

(2.5) devient

$$
\begin{gathered}
v(t, P)=\int_{U} G^{\prime}(t, P, Q) v_{0}(Q) d s^{\prime} d y, \quad t>0, \text { avec } P \in U, Q=\left(s^{\prime}, y\right), \\
\text { et } G^{\prime}(t, P, Q)=G(t, P, Q)\{g(P) g(Q)\}^{1 / 4} .
\end{gathered}
$$

$v(t, P)$ est une solution du problème de Cauchy $\left(\partial / \partial t+A^{\prime}\right) v(t, P)=0$, $v(0, P)=v_{0}(P)$, où $A^{\prime}$ est donné par

$$
\begin{aligned}
& \left(A^{\prime} v\right)(P)=g^{1 / 4} A\left(g^{-1 / 4} v\right)(P) \\
= & -\frac{\partial}{\partial x}\left(x \frac{\partial v}{\partial x}\right)-x \sum_{\alpha, \beta=1}^{n-1} g^{\alpha \beta}(P) \frac{\partial^{2} v}{\partial s_{\alpha} \partial s_{\beta}}+x \sum_{\alpha=1}^{n-1} b_{\alpha}(P) \frac{\partial v}{\partial s_{\alpha}}+b_{0}(P) v .
\end{aligned}
$$

Ainsi la recherche de $G$ est équivalente à celle de $G^{\prime}$ localement dans $U$ via la formule (2.7). Dès maintenant, nous écrivons toujours

$$
\begin{aligned}
& P=(s, x), \quad Q=\left(s^{\prime}, y\right), \quad R=\left(s^{\prime \prime}, z\right) \quad\left(s, s^{\prime}, s^{\prime \prime} \in R^{n-1} ; x, y, z \geqq 0\right), \\
& d P=d s d x, \quad d Q=d s^{\prime} d y \quad \text { et } \quad d R=d s^{\prime \prime} d z, \quad \text { si } \quad P, Q, R \in U,
\end{aligned}
$$


donc $d P, d Q$ et $d R$ sont les mesures de Lebesgue ordinaire dans $\boldsymbol{R}^{n}$.

Prenons un $Q \in U$, alors l'opérateur $A^{\prime}$ peut être approximé par $A_{Q}^{\prime}$ défini comme suit:

$$
A_{Q}^{\prime} v(P)=-\frac{\partial}{\partial x}\left(x \frac{\partial v}{\partial x}\right)-x \sum_{\alpha, \beta=1}^{n-1} g^{\alpha \beta}(Q) \frac{\partial^{2} v}{\partial s_{\alpha} \partial s_{\beta}}+x v .
$$

La différence $A^{\prime}-A_{Q}^{\prime}$ ne contient plus de dérivée par rapport à $x$ (l'Hypothèse III' y est essentielle):

$$
\begin{aligned}
\left(A^{\prime}-A_{Q}^{\prime}\right) v(P) & =x \sum_{\alpha, \beta=1}^{n-1}\left\{g^{\alpha \beta}(Q)-g^{\alpha \beta}(P)\right\} \frac{\partial^{2} v}{\partial s_{\alpha} \partial s_{\beta}}+x \sum_{\alpha=1}^{n-1} b_{\alpha}(P) \frac{\partial v}{\partial s_{\alpha}} \\
& +\left\{b_{0}(P)-x\right\} v .
\end{aligned}
$$

$Q$ étant fixe dans $\overline{\boldsymbol{R}_{+}^{n}}$, la solution unique du problème de Cauchy

$$
\left(\frac{\partial}{\partial t}+A_{Q}^{\prime}\right) w(t, P)=0, \quad t>0 \text { et } P \in \boldsymbol{R}_{+}^{n} ; \quad w(0, P)=w_{0}(P)
$$

est exprimée par

$$
w(t, P)=\int_{R_{+}^{n}} \Phi_{Q}(t, P, R) w_{0}(R) d R,
$$

où le noyau $\Phi_{Q}(t, P, R)$ est défini comme suit:

$$
\begin{aligned}
& \Phi_{Q}(t, P, R)=\Phi_{Q}\left(t, s-s^{\prime \prime}, x, z\right)=(2 \pi)^{1-n} \int_{R^{n-1}} e^{i\left(s-s^{\prime \prime}\right) \cdot \hat{s}} \hat{\Phi}_{Q}(t, \hat{s}, x, z) d \hat{s}, \\
& \text { avec } \hat{\Phi}_{Q}(t, \hat{s}, x, z)=\left.\hat{\Phi}(t, \sigma, x, z)\right|_{\sigma=\sigma_{Q}}, \\
& \hat{\Phi}(t, \sigma, x, z)=\frac{\sigma}{\operatorname{sh}(\sigma t)} I_{0}\left(\frac{2 \sigma \sqrt{x z}}{\operatorname{sh}(\sigma t)}\right) \exp \{-\sigma(x+z) \operatorname{coth}(\sigma t)\}, \\
& \text { et } \sigma_{Q}=\left\{\sum_{\alpha, \beta=1}^{n-1} g^{\alpha \beta}(Q) \hat{s}_{\alpha} \hat{s}_{\beta}+1\right\}^{1 / 2} ;
\end{aligned}
$$

où $I_{0}$ est la fonction de Bessel modifiée d'ordre 0 .

Cela posé, nous adoptons comme une paramétrix dans $U$ le noyau $\Phi_{Q}(t, P, Q)$. Pour estimer ceci et ses dérivées, nous introduisons une fonction auxiliaire

$$
\begin{aligned}
& E(t, P, Q ; \lambda, \mu, \nu ; c)=t^{\lambda-1 / 2}\left(A_{P Q}+t\right)^{\mu-1 / 2} \\
& \quad \times\left(B_{P Q}+\left|s-s^{\prime}\right|+t+\sqrt{t A_{P Q}}\right)^{\nu+1-n} \exp \left\{-c\left(t^{-1} B_{P Q}+\left|s-s^{\prime}\right|\right)\right\}, \\
& \text { où } A_{P Q}=(\sqrt{x}+\sqrt{y})^{2} \text { et } B_{P Q}=(\sqrt{x}-\sqrt{y})^{2} .
\end{aligned}
$$

Le lemme suivant a été démontré dans [8] (l'estimation de $(\partial / \partial x) \Phi_{R}$ est un peut améliorée que dans [8] car $\rho=-1$ dans le cas présent):

LEMme 2.1. Soient $0<t \leqq 1$ et $(P, Q, R) \in U \times U \times U$. Alors, nous avons 


$$
\begin{gathered}
\left|\left(\frac{\partial}{\partial s}\right)^{\kappa} \Phi_{R}(t, P, Q)\right| \leqq K E(t, P, Q ; 0,0,-|\kappa| ; c), s i|\kappa| \leqq 2 ; \\
\left|\frac{\partial}{\partial x} \Phi_{R}(t, P, Q)\right| \leqq K \sqrt{A_{P Q} / x} E(t, P, Q ;-1,0,0 ; c),
\end{gathered}
$$

avec des constantes $K$ et $c$ positives indépendantes des $(t, P, Q, R)$.

On ne reproduit pas ici la démonstration, les $K$ et $c$ dépendent essentiellement à la positivité de la matrice $\left(g^{\alpha \beta}(R)\right)$.

Comme $A^{\prime}-A_{Q}^{\prime}$ est de la forme (2.11), $\left|\left(A^{\prime}-A_{Q}^{\prime}\right) \Phi_{Q}(t, P, Q)\right|$ est plus petite que Cte. $E(t, P, Q ;-3 / 4,3 / 4,0 ; c)$, et donc, par une modification triviale, nous avons le

LEMmE 2.2. Soient $0<t \leqq 1$ et $(P, Q) \in U \times U$. Alors, nous avons

$$
\begin{aligned}
& \left|\left(A^{\prime}-A_{Q}^{\prime}\right) \Phi_{Q}(t, P, Q)\right| \\
& \quad \leqq K E\left(t, P, Q ;-\frac{3+\varepsilon}{4}, \frac{3-\varepsilon}{4}, \frac{\varepsilon}{2} ; c\right), \quad \text { pour } \quad 0 \leqq \varepsilon \leqq 1 .
\end{aligned}
$$

Nous aurons besoin aussi du

Lemme 2.3. Soint $0<t \leqq 1$ et $(P, Q) \in U \times U$. Alors, nous avons

$$
\left|\Phi_{Q}(t, P, Q)-\Phi_{P}(t, P, Q)\right| \leqq K E(t, P, Q ; 0,1 / 2,1 / 2 ; c) .
$$

Preuve DU Lemme 2.3. Comme $\Phi_{R}(t, P, Q)=\Phi_{R}(t, Q, P)$, on a

$$
\begin{aligned}
& \Phi_{Q}(t, P, Q)-\Phi_{P}(t, P, Q) \\
& \quad=(2 \pi)^{1-n} \int_{0}^{1} d \theta \int_{R^{n-1}} e^{i\left(s-s^{\prime}\right) \cdot \hat{s}} \frac{\partial \widehat{\Phi}}{\partial \sigma}(t, \sigma(\theta), x, y) \frac{\partial \sigma(\theta)}{\partial \theta} d \hat{s}
\end{aligned}
$$

avec $\sigma(\theta)=\sigma_{\theta Q+(1-\theta) P}$. On suit le même raisonnement que dans la démonstration du Lemme 2.1, et l'on a (2.17).

C.Q.F.D.

Maintenant, nous passons à la construction d'une paramétrix dans $\Omega$ tout entier. Soit $\left\{V_{i}, \xi_{i}(P)\right\}_{i=1}^{N}$ une partition d'unité finie suffisamment fine de la frontière $S$ : Chaque ouvert $V_{i}$ soit un voisinage de coordonnées locales $s^{(i)}=\left(s_{1}^{(i)}, s_{2}^{(i)}, \cdots, s_{n-1}^{(i)}\right)$ à l'origine $P_{i} \in V_{i}, \xi_{i}(P)$ soit une fonction de classe $C^{\infty}(S)$ à support compact dans $V_{i}$ tels que

$$
\bigcup_{i=1}^{N} V_{i}=S \text { et } \sum_{i=1}^{N} \xi_{i}(P) \equiv 1 \quad \text { sur } S \text {. }
$$

Définissons $U_{i}$ par $V_{i}$ comme (2.3). Alors, $\left\{U_{i}, \xi_{i}(P)\right\}_{i=1}^{N}$ est une partition d'unité de $\Omega_{\delta}$, où chaque $\xi_{i}(P)$ est considérée comme constante le long des géodésiques conormales à $S$ (c'est-à-dire, indépendante de la variable $x$ ). Notons par $\Phi_{Q}^{(i)}(t, P, Q)$ le noyau $\Phi_{Q}(t, P, Q)$ obtenu comme ci-dessus dans $U_{i}$ par rapport au système de coordonnées $\left(s^{(i)}, x\right)$. Alors, 


$$
\gamma(t, P, Q)=\sum_{i=1}^{N} \eta_{i}(P) \Phi_{Q}^{(i)}(t, P, Q) \xi_{i}(Q)\left\{g^{(i)}(P) g^{(i)}(Q)\right\}^{-1 / 4}
$$

est une paramétrix dans $\Omega_{\delta}$, où chaque $\eta_{i}(P)$ est une fonction de classe $C^{\infty}$ indépendante de $x$ (donc une fonction de $s^{(i)}$ seules) à support compact dans $U_{i}$ et vaut 1 sur un voisinage du support de $\xi_{i}(P)$, et $g^{(i)}(P)=$ $\operatorname{dét}\left(g^{\alpha \beta}\left(s^{(i)}, x\right)\right)^{-1}$.

D'autre part, nous regardons l'opérateur $A$ dans $\overline{\Omega_{\delta / 7}^{\prime}}$ par exemple (voir (2.1)). $A$ n'y est plus dégénéré, mais $A$ est uniformément fortement elliptique d'ordre 2. Soit $\bar{A}$ un opérateur uniformément fortement elliptique dans $\boldsymbol{R}^{n}$ tout entier (à coefficients constants en dehors de $\overline{\Omega_{\delta / 14}^{\prime}}$ ) qui est égal à $A$ dans un voisinage de $\overline{\Omega_{\delta / 7}^{\prime}}$. Nous faisons appel à l'aide de la solution fondamentale $\bar{\gamma}(t, P, Q)$ d'Eidelman (voir [2] p.23) dans $\boldsymbol{R}^{n}$ de $\partial / \partial t+\bar{A}$.

Alors, comme une paramétrix dans $\Omega$ de $\partial / \partial t+A$, nous employons le noyau suivant:

$$
G_{0}(t, P, Q)=\beta(P) \gamma(t, P, Q) \alpha(Q)+\bar{\beta}(P) \bar{\gamma}(t, P, Q) \bar{\alpha}(Q),
$$

où les $\alpha(P), \bar{\alpha}(P), \beta(P)$ et $\bar{\beta}(P)$ sont des fonctions de classe $C^{\infty}(\Omega)$ de $x$ seule ayant les propriétés suivantes:

$$
\begin{aligned}
& \alpha(P)=\left\{\begin{array}{lll}
1, & \operatorname{sur} \overline{\Omega_{3 \delta / 7}}, & \bar{\alpha}(P)=1-\alpha(P) ; \\
0, & \operatorname{sur} \overline{\Omega_{4 \delta / 7}^{\prime}} ;
\end{array}\right. \\
& \beta(P)=\left\{\begin{array}{lll}
1, & \operatorname{sur} \overline{\Omega_{\delta \delta / 7},} \\
0, & \operatorname{sur} \overline{\Omega_{6 \delta / 7}^{\prime}} ;
\end{array} \text { et } \bar{\beta}(P)=\left\{\begin{array}{lll}
1, & \text { sur } \overline{\Omega_{2 \delta / 7}^{\prime}} \\
0, & \text { sur } \overline{\Omega_{\delta / 7}}
\end{array}\right.\right.
\end{aligned}
$$

Donc, $\beta(P)($ resp. $\bar{\beta}(P))$ vaut 1 sur le $\delta / 7$-voisinage du support de $\alpha(P)($ resp. $\bar{\alpha}(P))$.

Nous pouvons maintenant chercher la fonction de Green $G(t, P, Q)$ en question sous la forme

$$
\begin{gathered}
G(t, P, Q)=G_{0}(t, P, Q)-\int_{0}^{t} d t^{\prime} \int_{\Omega} G_{0}\left(t-t^{\prime}, P, R\right) H\left(t^{\prime}, R, Q\right) d v_{R}, \\
\text { pour } 0<t \leqq 1 \text { et }(P, Q) \in \Omega \times \Omega,
\end{gathered}
$$

où $H(t, P, Q)$ est une fonction à choisir. Elle doit satisfaire à l'équation intégrale de Volterra

$$
\begin{aligned}
& H(t, P, Q)+\int_{0}^{t} d t^{\prime} \int_{\Omega} F\left(t-t^{\prime}, P, R\right) H\left(t^{\prime}, R, Q\right) d v_{R}=F(t, P, Q), \\
& \text { avec } F(t, P, Q)=\left(\frac{\partial}{\partial t}+A\right) G_{0}(t, P, Q) .
\end{aligned}
$$

Nous allons voir que (2.23) se résoud comme 


$$
\begin{gathered}
H(t, P, Q)=\sum_{k=1}^{\infty}(-1)^{k-1} F^{(k)}(t, P, Q), \quad \text { où } \quad F^{(1)}=F \\
\text { et } F^{(k)}(t, P, Q)=\int_{0}^{t} d t^{\prime} \int_{Q} F^{(k-1)}\left(t-t^{\prime}, P, R\right) F\left(t^{\prime}, R, Q\right) d v_{R} \\
\text { pour } \quad k=2,3, \cdots
\end{gathered}
$$

Nous effectuons l'approximation successive.

LEMME 2.4. Supposons que $0<t \leqq 1$ et que $P$ et $Q$ appartiennent à un $U_{i}$ commun. Alors, nous avons

$$
\begin{aligned}
& |F(t, P, Q)| \\
& \quad \leqq K E\left(t, P, Q ;-\frac{3+\varepsilon}{4}, \frac{3-\varepsilon}{4}, \frac{\varepsilon}{2} ; c\right), \quad \text { pour } \quad 0 \leqq \varepsilon \leqq 1,
\end{aligned}
$$

uniformément par rapport à $(t, P, Q)$.

Preuve. Récrivons $F(t, P, Q)$ en tenant compte de (2.14) et (2.20):

$$
\left\{\begin{array}{l}
F(t, P, Q)=\sum_{j=1}^{4} F_{j}(t, P, Q), \text { avec } \\
F_{1}(t, P, Q)=\sum_{i=1}^{N} \beta(P) \eta_{i}(P)\left\{\left(A^{\prime}-A_{Q}^{\prime}\right) \Phi_{Q}^{(i)}(t, P, Q)\right\} \alpha(Q) \xi_{i}(Q)\left(g_{P} g_{Q}\right)^{-1 / 4} \\
F_{2}(t, P, Q)=\sum_{i=1}^{N} \beta(P)\left\{\left[A^{\prime}, \eta_{i}(P) \cdot\right] \Phi_{Q}^{(i)}(t, P, Q)\right\} \alpha(Q) \xi_{i}(Q)\left(g_{P} g_{Q}\right)^{-1 / 4} \\
F_{3}(t, P, Q)=\sum_{i=1}^{N} \eta_{i}(P)\left\{\left[A^{\prime}, \beta(P) \cdot\right] \Phi_{Q}^{(i)}(t, P, Q)\right\} \alpha(Q) \xi_{i}(Q)\left(g_{P} g_{Q}\right)^{-1 / 4}, \\
\text { et } \quad F_{4}(t, P, Q)=\{[A, \bar{\beta}(P) \cdot] \bar{\gamma}(t, P, Q)\} \bar{\alpha}(Q),
\end{array}\right.
$$

où les $A^{\prime}$ et $A_{Q}^{\prime}$ sont définis par (2.8) et (2.10) respectivement dans $U_{i}$ au moyen du $\left(s^{(i)}, x\right)$, et $g_{P}$ signifie $g^{(i)}(P)$ dans (2.19).

D'abord, $F_{1}$ est majorée comme (2.25) grâce au Lemme 2.2. Pour $F_{2}$, le commutateur $\left[A^{\prime}, \eta_{i}(P) \cdot\right]$ est un opérateur différentiel d'ordre 1 seulement par rapport à $s^{(i)}$, donc par le Lemme 2.1, nous avons $\left|F_{2}\right| \leqq$ Cte. $E(t, P, Q ;-(2+\varepsilon) / 4,(3-\varepsilon) / 4, \varepsilon / 2 ; c)$. Ensuite pour $F_{3},\left[A^{\prime}, \beta(P) \cdot\right]$ est un opérateur différentiel d'ordre 1 seulement par rapport à $x$, et de plus, sur le support de $F_{3}, P$ et $Q$ s'éloignent de l'un de l'autre à $\delta / 7$ au moins. La situation est la même pour $F_{4}$. Douc, par le Lemme 2.1 et par une estimation de $\bar{\gamma}$ d'Eidelman (loc. cit.),

$$
\left|F_{3}(t, P, Q)+F_{4}(t, P, Q)\right| \leqq \text { Cte. } e^{-c^{\prime} / t}
$$

uniformément par rapport à $(t, P, Q)\left(c^{\prime}\right.$ dépend à $\delta$ mais elle est d'ordre $\delta^{2}$ au moins). En somme, nous avons le résultat. L'Hypothèse III' dans le paragraphe précédent est essentielle dans ce lemme. Notons que 
$F(t, P, Q)$ s'annule si $P$ n'est pas dans $\Omega_{\delta}$, et que les $F_{j}$ pour $1 \leqq j \leqq 3$ le font aussi s'il n'existe pas d'indice $i$ tel que $P$ et $Q$ appartiennent au même $U_{i}$.

C.Q.F.D.

Lemme 2.5. Soient $0<t \leqq 1$ et $(P, Q) \in \Omega \times \Omega$. La série de Neumann de (2.24) converge absolûment et uniformément, et l'on a

$$
|H(t, P, Q)| \leqq K E\left(t, P, Q ;-\frac{3+\varepsilon}{4}, \frac{3-\varepsilon}{4}, \frac{\varepsilon}{2} ; c\right)
$$

pour un $0<\varepsilon<1$ convenable.

Preuve. La fonction $E$ se prolonge pour tous $(P, Q) \in \Omega \times \Omega$ par une manière naturelle. Pour éviter de singularités logarithmiques, nous fixons un $0<\varepsilon<1$ irrationnel, et posons

$$
k_{0}=\operatorname{Max}\{k(\text { entier }) \geqq 1 ;(1-\varepsilon) k<2 \text { et } \varepsilon k<2(n-1)\} .
$$

Alors, en utilisant le Lemme 6.1, nous avons par récurrence sur $k$ l'inégalité suivante

$$
\begin{aligned}
& \left|F^{(k)}(t, P, Q)\right| \\
& \quad \leqq K_{k} E\left(t, P, Q ; \frac{1-\varepsilon}{4} k-1, \frac{3-\varepsilon}{4} k, \frac{\varepsilon k}{2} ; c\right) \text { pour } 1 \leqq k \leqq k_{0} .
\end{aligned}
$$

Posons provisoirement

$$
\begin{gathered}
\lambda_{k}=\frac{1-\varepsilon}{4} k-\frac{3}{2}, \mu_{k}=\frac{3-\varepsilon}{4} k-\frac{1}{2} \text { et } \rho_{k}=1-n+\frac{\varepsilon k}{2} \\
\text { pour } k=1,2,3, \cdots,
\end{gathered}
$$

et continuons l'itération de $F$ pour $k>k_{0}$. Choisissons $\varepsilon$ de sorte que

$$
\frac{n-1}{n}<\varepsilon<\frac{2 n-1}{2 n+1} \text {. }
$$

Alors, $k_{0}=2 n-1, \lambda_{2 n-1}<\lambda_{2 n}<-1<\lambda_{2 n+1}$ et $\rho_{2 n-1}<0<\rho_{2 n}$. Par un calcul analogue à ci-dessus, nous avons

$$
\begin{aligned}
& \left|F^{(2 n)}(t, P, Q)\right| \leqq K t^{\lambda_{2 n}}\left(A_{P Q}+t\right)^{\mu_{2 n}} \exp \left(-c B_{P Q} / t\right), \quad \text { et } \\
& \left|F^{(2 n+1)}(t, P, Q)\right| \leqq K t^{\lambda_{2 n+1}}\left(A_{P Q}+t\right)^{\mu_{2 n+1}} \exp \left(-c B_{P Q} / t\right) .
\end{aligned}
$$

Posons maintenent $F_{1}(t, P, Q)=F^{(2 n+1)}(t, P, Q), \bar{\lambda}_{1}=\lambda_{2 n+1}$ et $\bar{\mu}_{1}=\mu_{2 n+1}$, alors l'inégalité ci-dessus devient

$$
\begin{aligned}
& \left|F_{1}(t, P, Q)\right| \\
& \quad \leqq K t^{\bar{\lambda}_{1}}\left(A_{P Q}+t\right)^{\bar{\mu}_{1}} \exp \left(-c B_{P Q} / t\right) \text {, où } \bar{\lambda}_{1}>-1 \text { et } \bar{\mu}_{1}>1 .
\end{aligned}
$$

Soit $F_{1}^{(k)}$ l'itéré de $k$ fois de $F_{1}=F_{1}^{(1)}$. Si l'on applique la Proposition 4.1, (F) et le Lemme 5.1, on a 


$$
\left|F_{1}^{\prime k)}(t, P, Q)\right| \leqq K_{k} t^{\bar{\lambda}_{k}}\left(A_{P Q}+t\right)^{\bar{\mu}_{k}} \exp \left(-c B_{P Q} / t\right), k=1,2,3, \cdots,
$$

où $\bar{\lambda}_{k}=k\left(3 / 2+\bar{\lambda}_{1}\right)-3 / 2$ et $\bar{\mu}_{k}=k\left(1 / 2+\bar{\mu}_{1}\right)-1 / 2$. Cette (2.33) nous fait apprendre que, si $k$ est suffisamment grand, $F_{1}^{(k)}=F^{((2 n+1) k)}$ est bornée. Et donc la série de (2.24) converge absolûment et uniformément et nous obtenons (2.27).

C.Q.F.D.

La fonction $G(t, P, Q)$ définie par (2.22) avec cette $H(t, P, Q)$ est précisément la fonction de Green pour $\partial / \partial t+A$. Pour le voir, il suffit de vérifier que, pour toute $u_{0}(P)$ suffisamment régulière,

$$
u(t, P)=\int_{\Omega} G(t, P, Q) u_{0}(Q) d v_{Q}
$$

est une solution suffisamment régulière du problème de Cauchy $(\partial / \partial t+$ A) $u(t, P)=0, t>0$ et $u(0, P)=u_{0}(P)$. Alors, par l'unicité des solutions, nous voyons que $u(t, P)=\left(e^{-t A} u_{0}\right)(P)$. Donc, $G(t, P, Q)$ représente le semigroupe $e^{-t A}$. La démonstration détaillée est ommise.

3. Démonstration du Théorème 1.3. A la fin du $\S 1$, nous avons réduit la considération de la $\zeta$-fonction d'Epstein $Z(\alpha)$ à celle de $Z_{0}(\alpha)$ définie par (1.21).

Par (2.22), nous avons

$$
\begin{aligned}
& G(t, P, P)=G_{0}(t, P, P)-G^{\prime}(t, P, P), \\
& \text { où } \quad G^{\prime}(t, P, P)=\int_{0}^{t} d t^{\prime} \int_{\Omega} G_{0}\left(t-t^{\prime}, P, R\right) H\left(t^{\prime}, R, P\right) d v_{R} .
\end{aligned}
$$

Lemme 3.1. Nous avons, pout $0<t \leqq 1$ et $P \in \Omega_{\delta / 7}$,

$$
\left|G^{\prime}(t, P, P)\right| \leqq \text { Cte. } t^{(1 / 4)-(n / 2)}(x+t)^{(3 / 4)-(n / 2)} .
$$

Preuve. Comme nous avons vu,

$$
\begin{aligned}
\left|G_{0}\left(t-t^{\prime}, P, R\right)\right| & \leqq \text { Cte. } E\left(t-t^{\prime}, P, R ; 0,0,0 ; c\right) \\
& \leqq \text { Cte. } E\left(t-t^{\prime}, P, R ;-\varepsilon / 4,-\varepsilon / 4, \varepsilon / 2 ; c\right), \text { et } \\
\left|H\left(t^{\prime}, R, P\right)\right| & \leqq \text { Cte. } E\left(t^{\prime}, R, P ;-(3+\varepsilon) / 4,(3-\varepsilon) / 4, \varepsilon / 2 ; c\right)
\end{aligned}
$$

avec un $0<\varepsilon<1$. Donc, par le Lemme 6.2 avec

$$
\lambda=-\varepsilon / 4, \mu=-\varepsilon / 4, \nu=\varepsilon / 2, \lambda^{\prime}=-(3+\varepsilon) / 4, \mu^{\prime}=(3-\varepsilon) / 4 \text { et } \nu^{\prime}=\varepsilon / 2,
$$

nous obtenons (3.2).

C.Q.F.D.

COROLLAIRE 3.1. La fonction $\int_{0}^{1} t^{\alpha-1} d t \int_{\delta_{\delta / 7}} G^{\prime}(t, P, P) d v_{P}$ est holomorphe dans $\operatorname{Re} \alpha>3 / 4$ si $n=2$, dans $\operatorname{Re} \alpha>5 / 4$ si $n=3$ et dans $\operatorname{Re} \alpha>n-2$ si $n \geqq 4$.

Ainsi, la recherche de $Z(\alpha)$ est réduite à celle de 


$$
Z_{00}(\alpha)=\frac{1}{\Gamma(\alpha)} \int_{0}^{1} t^{\alpha-1} d t \int_{\Omega_{\delta / 7}} G_{0}(t, P, P) d v_{P} .
$$

Maintenant, nous nous rappelons de la structure riemannienne sur $\bar{\Omega}$ induite par la métrique $d l$. En utilisant l'application $P \rightarrow \bar{P}$ expliquée au début du $\S 2$, nous pouvons écrire

$$
d v_{P}=m(P) d S_{\bar{P}} d x,
$$

où $d S_{\bar{P}}$ est l'élément volumique induit par la métrique $d l$ sur $S$ et $m(P)$ est une densité positive telle que $m(P)=1$ sur $S$. Donc, (3.3) s'écrit encore

$$
\begin{aligned}
& \Gamma(\alpha) Z_{00}(\alpha)=\int_{0}^{1} t^{\alpha-1} d t \int_{s}\left\{k(t, \bar{P})+k^{\prime}(t, \bar{P})\right\} d S_{\bar{P}}, \\
& \text { avec } k(t, \bar{P})=\int_{L(\bar{P})} G_{0}(t, P, P) d x \\
& \text { et } k^{\prime}(t, \bar{P})=\int_{L(\bar{P})} G_{0}(t, P, P)\{m(P)-1\} d x,
\end{aligned}
$$

où $L(\bar{P})$ désigne la ligne géodésique du longueur $\delta / 7$ issue de $\bar{P} \in S$ à la direction conormale.

Lemme 3.2. Soit $P \in \Omega_{\delta / 7}$. Alors, $G_{0}(t, P, P)$ est une fonction de $(t, x)$ seules et égale à $\gamma(t ; x)$ définie par (7.1) et (7.2).

Preuve. Avec les notations de (2.19) et (2.20), nous avons

$$
G_{0}(t, P, P)=\sum_{i=1}^{N} \xi_{i}(\bar{P}) \Phi_{P}^{(i)}(t, P, P) / \sqrt{g^{(i)}(P)}, \quad \text { si } \quad P \in \Omega_{\delta / 7} .
$$

Si donc on voit que

$$
\Phi_{P}^{(i)}(t, P, P) / \sqrt{g^{(i)}(P)}=\gamma(t ; x) \quad \text { pour } \quad P \in U_{i} \cap \Omega_{\delta / 7},
$$

il vient $G_{0}(t, P, P)=\gamma(t ; x)$, car $\sum \xi_{i}(\bar{P})=1$. Mais (3.6) se voit facilement si l'on effectue dans (2.12) la transformation affine $\hat{s}=T_{P}^{-1 / 2} \hat{s}^{\prime}$ où $T_{P}=\left(g^{\alpha \beta}(P)\right)_{\alpha, \beta=1}^{n-1}$.

C.Q.F.D.

Lemme 3.3. Soit $n \geqq 3$. Alors, lorsque $t \downarrow 0$, nous avons

$$
\begin{gathered}
t^{n-1} k(t, \bar{P})=(n-2) !\left(A_{n} /|S|\right)+O(t), \quad e t, \\
t^{n-1} k^{\prime}(t, \bar{P})=O(\sqrt{t}),
\end{gathered}
$$

où $A_{n}$ est celle définie dans le Théorème 1.3.

Preuve. Considérons la différence entre $k(t, \bar{P})$ et $k(t)$ définie par (7.2). Comme $|\gamma(t ; x)| \leqq$ Cte. $\{t(x+t)\}^{-n / 2}$, on a $|k(t, \bar{P})-\kappa(t)|=O\left(t^{2-n}\right)$, d'où (3.7), car $\left\{t^{n-1} \kappa(t)\right\}_{t=0}=(n-2) ! A_{n} /|S|$ d'après la Proposition 7.1. 
Ensuite, $\left|G_{0}(t, P, P)\{m(P)-1\}\right| \leqq$ Cte. $x\{t(x+t)\}^{-n / 2}$, car $m(\bar{P})=1$. Donc, $\left|k^{\prime}(t, \bar{P})\right|$ est $O\left(t^{-3 / 2}\right)$ si $n=3, O\left(t^{-2}(1+\log (1 / t))\right)$ si $n=4$ et $O\left(t^{2-n}\right)$ si $n \geqq 5$.

C.Q.F.D.

Lemme 3.4. Soit $n=2$. Alors, lorsque $t \downarrow 0$, nous avons

$$
\begin{gathered}
t k(t, \bar{P})=\frac{1}{4 \pi} \log \frac{1}{t}+\frac{1}{4 \pi}\{\log (16 \delta / 7)+\gamma\}+O(\sqrt{t}), \quad e t, \\
t k^{\prime}(t, \bar{P})=\frac{1}{4 \pi} \int_{0}^{\delta / 7} \frac{m(P)-1}{x} d x+O(\sqrt{t}) .
\end{gathered}
$$

Preuve. $k(t, \bar{P})$ n'est autre que $\kappa_{(0)}(t ; 2 \delta / 7)$ définie par $(7.3)$, d'où (3.9) d'après la Proposition 7.2 et le Corollaire 7.4. Ensuite, $k^{\prime}(t, \bar{P})$ est précisément l'intégrale traitée dans la Proposition 7.3 avec $a(x)=m(P)-1$ et $\delta$ remplacé par $\delta / 7$, d'où (3.10) par le Corollaire 7.4.

C.Q.F.D.

Fin de la démonstration du Théorème 1.3:

Par (3.5), nous pouvons écrire

$$
\begin{aligned}
\Gamma(\alpha) Z(\alpha)= & \int_{0}^{1} t^{\alpha-1} d t \int_{S} k(t, \bar{P}) d S_{\bar{P}}+\int_{0}^{1} t^{\alpha-1} d t \int_{S} k^{\prime}(t, \bar{P}) d S_{\bar{P}} \\
& +\Gamma(\alpha)\left\{Z_{0}(\alpha)-Z_{00}(\alpha)\right\}+\Gamma(\alpha)\left\{Z(\alpha)-Z_{0}(\alpha)\right\} .
\end{aligned}
$$

Soit premièrement $n \geqq 3$ : Alors, chaque terme à droite de (3.11) sauf le premier est holomorphe dans $\operatorname{Re} \alpha>n-(3 / 2)$ par les Lemmes $1.1,1.2,3.3$ et le Corollaire 3.1. Et le premier est égal à $(n-2) ! A_{n}(\alpha-$ $n+1)^{-1}+$ (une fonction holomorphe dans $\operatorname{Re} \alpha>n-2$ ), d'après le Lemme 3.3. D'où la partie (ii) du Théorème 1.3.

Soit ensuite $n=2$ : Le premier terme à droite de (3.11) est

$$
\begin{aligned}
& \frac{|S|}{4 \pi}(\alpha-1)^{-2}+\frac{|S|}{4 \pi}\{\log (16 \delta / 7)+\gamma\}(\alpha-1)^{-1} \\
& \quad+\text { (une fonction holomorphe dans } \operatorname{Re} \alpha>1 / 2) .
\end{aligned}
$$

Le deuxième est égal à

$$
\begin{aligned}
& \frac{1}{4 \pi} \int_{S} d S_{\bar{P}} \int_{0}^{\delta / 7} \frac{m(P)-1}{x} d x(\alpha-1)^{-1} \\
& \quad+\text { (une fonction holomorphe dans } \operatorname{Re} \alpha>1 / 2) .
\end{aligned}
$$

Le troisième est déjà holomorphe dans $\operatorname{Re} \alpha>3 / 4$. Et le dernier terme à droite de (3.11) est égal à

$$
\begin{aligned}
& \frac{1}{4 \pi} \int_{\Omega^{\prime} / / 7} \frac{1}{\phi(P)} d v_{P}(\alpha-1)^{-1} \\
& \quad+(\text { une fonction holomorphe dans } \operatorname{Re} \alpha>0),
\end{aligned}
$$


d'après le Lemme 1.2. En somme, nous avons

$$
\begin{aligned}
\Gamma(\alpha) Z(\alpha)= & \frac{|S|}{4 \pi}(\alpha-1)^{-2}+\frac{M}{4 \pi}(\alpha-1)^{-1} \\
& +(\text { une fonction holomorphe dans } \operatorname{Re} \alpha>3 / 4) .
\end{aligned}
$$

Calculons maintenant le coefficient $M$.

$$
\begin{aligned}
M-|S|\{4 \log 2 & +\gamma\}=|S| \log (\delta / 7) \\
& +\int_{S} d S_{\bar{P}}^{-} \int_{0}^{\delta / 7} \frac{m(P)-1}{x} d x+\int_{\Omega_{\delta^{\prime} / 7}} \frac{1}{\phi(P)} d v_{P} .
\end{aligned}
$$

Le membre à droite de (3.13) est égal à

$$
|S| \log b+\int_{S} d S_{\bar{P}} \int_{0}^{b} \frac{m(P)-1}{x} d x+\int_{\Omega_{b}^{\prime}} \frac{1}{\phi(P)} d v_{P},
$$

quel que soit $0<b<\delta$. Ceci est donc un choix de la partie finie de l'intégrale $\int_{\Omega} \phi(P)^{-1} d v_{P}$ qui est divergente. Nous pouvons l'écrire aussi

$$
P f . \int_{0}^{\delta}\left\{\int_{S} m(P) d S_{\bar{P}}\right\} \frac{d x}{x}+\int_{\Omega_{\delta}^{\prime}} \phi(P)^{-1} d v_{P},
$$

où la dernière $P f$. est prise pour l'intégrale par rapport à $x$ au sens de Schwartz [6].

Le Théorème 1.3. est maintenant établi.

4. Intégrales dépendantes aux paramètres. Le but de ce paragraphe est d'estimer l'intégrale suivante:

$$
\begin{aligned}
& I=I(A, B, P ; \alpha, \beta, \rho) \\
& =\int_{0}^{1} t^{\alpha}(1-t)^{\beta}(P+t)^{-\rho} \exp \left(-\frac{A}{t}-\frac{B}{1-t}\right) d t,
\end{aligned}
$$

où $A>0, B>0, P>0, \alpha \in \boldsymbol{R}, \beta \in \boldsymbol{R}$ et $\rho \in \boldsymbol{R}$ sont des paramètres. Supposons de plus que

$$
(\alpha+1)(\alpha-\rho+1)(\beta+1) \neq 0 \text { et que } \rho \geqq 0 .
$$

Posons

$$
\begin{aligned}
& C=(\sqrt{A}+\sqrt{B})^{2}, \quad D=1+\sqrt{A C}, \quad E=1+\sqrt{B C} \\
& \text { et } F=1+\sqrt{A B} .
\end{aligned}
$$

Alors, le résultat se résume comme la proposition suivante:

Proposition 4.1. Sous la restriction (4.2), nous avons les inégalités suivantes: 
(A) Si $\alpha<-1$ et $\beta<-1$,

$$
e^{c} I \leqq \text { Cte. }\left\{\left(P+\frac{A}{D}\right)^{-\rho} \frac{(A / D)^{\alpha+1}}{\sqrt{\bar{D}}}+(P+1)^{-\rho} \frac{(B / E)^{\beta+1}}{\sqrt{\bar{E}}}\right\} ;
$$

(B) Si $\alpha<-1$ et $\beta>-1$,

$$
e^{C} I \leqq \text { Cte. }\left(P+\frac{A}{D}\right)^{-\rho}\left(\frac{A}{D}\right)^{\alpha+1}\left(\frac{E}{C+1}\right)^{\beta+1} / \sqrt{F} ;
$$

(C) Si $-1<\alpha<\rho-1$ et $\beta<-1$,

(4.6) $\quad e^{c} I \leqq$ Cte. $\left\{\left(P+\frac{A}{D}\right)^{-\rho}\left(\frac{D P+A}{(C+1) P+D}\right)^{\alpha+1} \frac{1}{\sqrt{D}}+(P+1)^{-\rho} \frac{(B / E)^{\beta+1}}{\sqrt{E}}\right\}$;

(D) $S i-1<\alpha<\rho-1$ et $\beta>-1$

$$
e^{c} I \leqq \text { Cte. }\left(P+\frac{A}{D}\right)^{-\rho}\left(\frac{D P+A}{(C+1) P+D}\right)^{\alpha+1}\left(\frac{E}{C+1}\right)^{\beta+1} / \sqrt{F} ;
$$

(E) Si $\alpha>\rho-1$ et $\beta<-1$,

$$
e^{C} I \leqq \text { Cte. }\left(P+\frac{D}{C+1}\right)^{-\rho}\left(\frac{D}{C+1}\right)^{\alpha+1}\left(\frac{B}{E}\right)^{\beta+1} / \sqrt{F} ;
$$

(F) Si $\alpha>\rho-1$ et $\beta>-1$,

$$
e^{C} I \leqq \text { Cte. }\left(P+\frac{D}{C+1}\right)^{-\rho}\left(\frac{D}{C+1}\right)^{\alpha+1}\left(\frac{E}{C+1}\right)^{\beta+1} / \sqrt{F} ;
$$

Les constantes dépendent aux $(\alpha, \beta, \rho)$ mais elles sont indépendantes des $(A, B, P)$.

Posons d'abord

$$
a=\sqrt{A / C}, \quad b=\sqrt{B / C} \quad \text { et } s=\frac{a^{2}}{t}+\frac{b^{2}}{1-t}-1,
$$

donc $0<a, b<1$ et $a+b=1$. Lorsque $t$ varie de 0 à 1 , la fonction $s$ atteint son minimum 0 à $t=a$, et elle prend chaque valeur positive exactement deux fois. Résolvons la dernière équation de (4.10) par rapport à $t$, elle admet les deux racines $t_{1}(s)$ et $t_{2}(s)$ :

$$
\begin{aligned}
& t_{1}(s)=h(s) /(s+1) \text { et } t_{2}(s)=a^{2} / h(s), \\
& \text { où } h(s)=a+(s+D(s)) / 2 \text { et } D(s)=\sqrt{s(s+4 a b)} .
\end{aligned}
$$

Alors, $t_{1}(0)=t_{2}(0)=a$ et $0<t_{2}(s)<a<t_{1}(s)<1$ si $s>0$. Nous écrivons $I$ comme la somme de deux intégrales par rapport à $s$ :

$$
e^{c} I=J=J_{1}+J_{2} \text { avec }
$$

$$
J_{j}=\int_{0}^{\infty} t_{j}(s)^{\alpha+1}\left\{1-t_{j}(s)\right\}^{\beta+1}\left\{P+t_{j}(s)\right\}^{-\rho} \frac{e^{-C s}}{D(s)} d s, \quad j=1,2,
$$


où nous avons tenu compte de la relation $d t /\{t(1-t)\}=\mp d s / D(s)$ suivant $0<t<a$ ou $a<t<1$.

Dès maintenant, nous écrivons $g_{1} \sim g_{2}$ (ou $g_{1} \precsim g_{2}$ ) pour les deux fonctions $g_{j}=g_{j}(A, B, \cdots ; a, b, \cdots, s, \cdots ; a, \beta, \cdots)$ positives quand il existe des constantes $K_{1}$ et $K_{2}$ (resp. $K_{3}$ ) positives telles que

$$
\left.K_{1} \leqq g_{1} / g_{2} \leqq K_{2} \quad \text { (resp. } g_{1} \leqq K_{3} g_{2}\right) \text { ， }
$$

où les $K_{1}$ et $K_{2}$ (resp. $K_{3}$ ) peuvent être dépendantes aux quantités en caractères grees $(\alpha, \beta, \cdots)$ mais elles doivent être indépendantes des quantités en caractères latins $(A, B, \cdots ; a, b, \cdots, s, \cdots)$. Ainsi, les notations et $\lesssim$ signifient l'équivalence et majoration en ignorant des constantes.

Nous introduisons de quelques fonctions auxiliaires:

$$
\begin{aligned}
& \Psi(\alpha, \gamma ; x) \\
= & \frac{1}{\Gamma(\alpha)} \int_{0}^{\infty} t^{\alpha-1}(t+1)^{\gamma-\alpha-1} e^{-\alpha t} d t, \text { pour } \alpha>0, \gamma \in \boldsymbol{R} \text { et } x>0 ;
\end{aligned}
$$

$$
F(C, Q ; \alpha)=\int_{1}^{Q} s^{\alpha} e^{-C s} d s, \text { pour } C>0, Q \geqq 1 \text { et } \alpha \in \boldsymbol{R} ;
$$

$$
G(C, p ; \alpha, \beta)=\int_{0}^{\infty}(s+p)^{\alpha+(1 / 2)}(s+1)^{-\alpha-\beta-2} \frac{e^{-C s}}{\sqrt{s}} d s,
$$

pour $C>0,0<p \leqq 1, \alpha \in \boldsymbol{R}$ et $\beta \in \boldsymbol{R}$;

$$
\begin{gathered}
H(C, p, q ; \alpha, \beta, \gamma)=\int_{0}^{\infty}(s+p)^{\alpha+(1 / 2)}(s+q)^{-\beta}(s+1)^{\beta-\alpha-\gamma-2} \frac{e^{-C s}}{\sqrt{s}} d s, \\
\text { pour } C>0, \quad 0<p \leqq q \leqq 1, \alpha \in \boldsymbol{R}, \quad \beta \in \boldsymbol{R} \text { et } \gamma \in \boldsymbol{R} .
\end{gathered}
$$

Nous allons comparer les $J_{1}$ et $J_{2}$ avec ces fonctions.

Lemme 4.1. (1) Soit $0<a \leqq 1 / 2$. Alors,

$$
\begin{aligned}
& J_{1} \sim(P+1)^{-\rho} H\left(C, a, \frac{P+a}{P+1} ; \alpha, \rho, \beta\right), \text { et } \\
& J_{2} \sim P^{-\rho}\left(\frac{P a}{P+a}\right)^{\alpha+1} G\left(\frac{C a(P+a)}{P}, \frac{P}{P+a} ; \rho-\alpha-2, \alpha\right) ;
\end{aligned}
$$

(2) Soit par contre $1 / 2 \leqq a<1$. Alors,

$$
\begin{aligned}
& J_{1} \sim(P+1)^{-\rho} b^{\beta+1} \Psi(1 / 2,-\beta ; C b), \quad \text { et } \\
& J_{2} \sim P^{-\rho}\left(\frac{P}{P+1}\right)^{\alpha+1} H\left(\frac{C(P+1)}{P}, \frac{P b}{P+1}, \frac{P}{P+1} ; \beta, \alpha+\beta+2-\rho, \alpha\right) .
\end{aligned}
$$

Les équivalences sont uniformes par rapport $\grave{a}(C, P, a, b)$ chaque fois $q u$ 'on fixe les $(\alpha, \beta, \rho)$. 
PReUve. Nous voyons facilement par (4.11) que

$$
\begin{gathered}
t_{1}(s) \sim \frac{s+a}{s+1}, 1-t_{1}(s) \sim \frac{b^{2}}{s+b} ; t_{2}(s) \sim \frac{a^{2}}{s+a}, 1-t_{2}(s) \sim \frac{s+b}{s+1} ; \\
\text { et que } D(s) \sim \sqrt{s(s+a b)} .
\end{gathered}
$$

Done nous avons

$$
\begin{gathered}
(P+1)^{\rho} J_{1} \sim b^{2 \beta+2} \int_{0}^{\infty}(s+a)^{\alpha+1}(s+b)^{-\beta-1}(s+1)^{\rho-\alpha-1}\left(s+\frac{P+a}{P+1}\right)^{-\rho} \frac{e^{-C s} d s}{\sqrt{s(s+a b)}}, \\
P^{\rho} J_{2} \sim a^{2 \alpha+2} \int_{0}^{\infty}(s+a)^{\rho-\alpha-1}(s+b)^{\beta+1}(s+1)^{-\beta-1}\left(s+a+\frac{a^{2}}{P}\right)^{-\rho} \frac{e^{-c s} d s}{\sqrt{s(s+a b)}} .
\end{gathered}
$$

Si $0<a \leqq 1 / 2$, alors $s+a b \sim s+a, s+b \sim s+1$. Si $1 / 2 \leqq a<1$, alors $s+a b \sim s+b, s+a \sim s+(P+a) /(P+1) \sim s+1$. D'où (4.17) et (4.18).

C.Q.F.D.

Ainsi, l'estimation de $J$ est réduite à celle des fonctions auxiliaires.

Lemme 4.2. Soit $\gamma \neq 1$ et $\alpha>0$. Alors

$$
\Psi(\alpha, \gamma ; x) \sim\left(\frac{x}{x+1}\right)^{\operatorname{Min}(1-\gamma, 0)}(x+1)^{-\alpha}, \text { pour } x>0,
$$

l'équivalence étant uniforme par rapport à $x$ pour les $(\alpha, \gamma)$ fixes.

Pour la démonstration, voir [3], Vol. 1, Chap. 6.

LEMmE 4.3. Soit $\alpha \neq-1$. Alors, pour $C>0$ et $Q \geqq 1$,

$$
F(C, Q ; \alpha) \precsim e^{-c} \Psi\left(1, \alpha+2 ; C+\frac{1}{Q}\right),
$$

la majoration étant uniforme par rapport à $(C, Q)$ pour $\alpha$ fixe. donc

PREUve. Soit d'abord $C Q \leqq 1$. Alors $e^{-C s} \sim 1$ pour $1 \leqq s \leqq Q$, et

$$
F \sim \int_{1}^{Q} s^{\alpha} d s \sim Q^{\operatorname{Max}(\alpha+1,0)} \sim \Psi(1, \alpha+2 ; 1 / Q) .
$$

Soit ensuite $C Q \geqq 1$. Alors

$$
C^{\alpha+1} F<\int_{C}^{\infty} s^{\alpha} e^{-s} d s=e^{-c} C^{\alpha+1} \Psi(1, \alpha+2 ; C) .
$$

LEMME 4.4. Soit $(\alpha+1)(\beta+1) \neq 0$. Alors, pour $C>0$ et $0<p \leqq 1$, (4.21) $G(C, p ; \alpha, \beta) \sim p^{\alpha+1} \Psi(1 / 2, \alpha+2 ;(C+1) p)+e^{-c} \Psi(1,-\beta ; C)$, uniformément par rapport à $(C, p)$ pour les $(\alpha, \beta)$ fixes.

Preuve. Partageons l'intégrale de (4.15) en deux parties: $G=$ $\int_{0}^{1}+\int_{1}^{\infty} \equiv G_{1}+G_{2}$. D'abord, $G_{2} \sim e^{-c} \Psi(1,-\beta ; C)$. Pour $G_{1}$, soit première- 
ment $C \leqq 1$. Alors, $G_{1} \sim p^{\min (\alpha+1,0)} \sim p^{\alpha+1} \Psi(1 / 2, \alpha+2 ; p)$.

Soit ensuite $C \geqq 1$. Alors,

$$
G_{1} \sim p^{\alpha+1} \int_{0}^{1 / p}(s+1)^{\alpha+(1 / 2)} \frac{e^{-C p s}}{\sqrt{s}} d s \sim p^{\alpha+1} \Psi(1 / 2, \alpha+2 ; C p) \text {. C.Q.F.D. }
$$

Lemme 4.5. Soit $(\alpha+1)(\alpha-\beta+1)(\gamma+1) \neq 0$. Alors, pour $C>0$ et $0<p \leqq q \leqq 1$, nous avons

$$
\begin{gathered}
H(C, p, q ; \alpha, \beta, \gamma) \preccurlyeq p^{\alpha+1} q^{-\beta}\left\{\frac{1}{\sqrt{C p+1}}+e^{-c p} \Psi\left(1, \alpha+2 ;\left(C+\frac{1}{q}\right) p\right)\right\} \\
\quad+q^{\alpha+1-\beta} e^{-C q} \Psi(1, \alpha-\beta+2 ;(C+1) q)+e^{-C} \Psi(1,-\gamma ; C),
\end{gathered}
$$

uniformément par rapport à $(C, p, q)$ pour les $(\alpha, \beta, \gamma)$ fixes.

Preuve. Partageons l'intégrale de (4.16) en quatre parties:

$$
H=\int_{0}^{p}+\int_{p}^{q}+\int_{q}^{1}+\int_{1}^{\infty} \equiv H_{1}+H_{2}+H_{3}+H_{4} .
$$

Alors, $H_{1} \sim p^{\alpha+1} q^{-\beta} / \sqrt{C p+1}, \quad H_{2} \sim p^{\alpha+1} q^{-\beta} F(C p, q / p ; \alpha), H_{3} \sim q^{\alpha+1-\beta} F(C q$, $1 / q ; \alpha-\beta)$ et $H_{4} \sim e^{-c} \Psi(1,-\gamma ; C)$, d'où (4.22) d'après le Lemme 4.3.

C.Q.F.D.

CorollaIRE 4.4. Sous les hypothèses du Lemme 4.4, nous avons les suivantes:

$$
\begin{aligned}
& \left\{\begin{array}{c}
\left(1^{\circ}\right) \quad \text { Si } \alpha+1>0 \text { et } \beta+1>0, \\
G(C, p ; \alpha, \beta) \precsim\left(\frac{C p+1}{C+1}\right)^{\alpha+1} / \sqrt{C p+1} ; \\
\left(2^{\circ}\right) \quad \text { Si } \alpha+1>0 \text { et } \beta+1<0, \\
G \precsim\left(\frac{C}{C+1}\right)^{\beta+1}\left(\frac{C p+1}{C+1}\right)^{\alpha+1} / \sqrt{C p+1} ; \\
\left(3^{\circ}\right) \text { Si } \alpha+1<0 \text { et } \beta+1>0, \quad G \precsim p^{\alpha+1} / \sqrt{C p+1} ;
\end{array}\right. \\
& \left(^{\circ}\right) \text { Si } \alpha+1<0 \text { et } \beta+1<0 \text {, } \\
& G \preccurlyeq\left(p^{\alpha+1} / \sqrt{C p+1}\right)+\left(\frac{C}{C+1}\right)^{\beta+1} \frac{e^{-C}}{C+1} .
\end{aligned}
$$

Preuve. Dans chaque cas $\left(1^{\circ}\right) \sim\left(4^{\circ}\right)$, on estime le membre à droite de (4.21) en distinguant les cas $0<C \leqq 1,1 \leqq C \leqq 1 / p$ et $C \geqq 1 / p$.

C.Q.F.D.

CoRollaire 4.5. Dans le Lemme 4.5, nous distinguons les 8 cas suivant les signes des $\alpha+1, \alpha-\beta+1$ et $\gamma+1$. Nous écrivons, par exemple, 
$(+++)$ pour exprimer le cas où $\alpha+1>0, \alpha-\beta+1>0$ et $\gamma+1>0$. Alors, nous avons les suivantes:

$$
\begin{aligned}
& \left(5^{\circ}\right)(+++) \\
& H(C, p, q ; \alpha, \beta, \gamma) \precsim\left(\frac{C p+1}{C+1}\right)^{\alpha+1}\left(\frac{C q+1}{C+1}\right)^{-\beta} / \sqrt{C p+1}, \\
& \left(^{\circ}\right) \quad(++-) \quad H \precsim\left(\frac{C p+1}{C+1}\right)^{\alpha+1}\left(\frac{C q+1}{C+1}\right)^{-\beta}\left(\frac{C}{C+1}\right)^{\gamma+1} / \sqrt{C p+1} \text {; } \\
& \left(7^{\circ}\right) \quad(+-+) \quad H \precsim q^{\alpha-\beta+1}\left(\frac{C p+1}{C q+1}\right)^{\alpha+1} / \sqrt{C p+1} \text {; } \\
& \left(8^{\circ}\right)(+--) \\
& H \precsim\left\{q^{\alpha-\beta+1}\left(\frac{C p+1}{C q+1}\right)^{\alpha+1} / \sqrt{C p+1}\right\}+\left(\frac{C}{C+1}\right)^{\gamma+1} \frac{e^{-C}}{C+1} ; \\
& \left(9^{\circ}\right) \quad(-++) \quad H \precsim\left\{p^{\alpha+1} q^{-\beta} / \sqrt{C p+1}+(C+1)^{\beta-\alpha-1} e^{-C q}\right. \text {; } \\
& \left(10^{\circ}\right)(-+-) \\
& H \precsim\left\{p^{\alpha+1} q^{-\beta} / \sqrt{C p+1}\right\}+(C+1)^{\beta-\alpha-1}\left(\frac{C}{C+1}\right)^{\gamma+1} e^{-C q} ; \\
& \left(11^{\circ}\right)(--+) \quad H \precsim p^{\alpha+1} q^{-\beta} / \sqrt{C p+1} \text {; } \\
& \left(12^{\circ}\right)(---) H \precsim\left\{p^{\alpha+1} q^{-\beta} / \sqrt{C p+1}\right\}+\left(\frac{C}{C+1}\right)^{\gamma+1} \frac{e^{-C}}{C+1} \text {. }
\end{aligned}
$$

Les majorations sont uniformes par rapport aux $(C, p, q)$ quand on fixe les $(\alpha, \beta, \gamma)$.

Preuve. On estime le membre à droite de (4.22) suivant les cas $0<C \leqq 1,1 \leqq C \leqq 1 / q, 1 / q \leqq C \leqq 1 / p$ et $C \geqq 1 / p$.

C.Q.F.D.

Démonstration de la Proposition 4.1: Nous avons vu dans le Lemme 4.1 que les $J_{1}$ et $J_{2}$ sont majorées par les $\Psi, G$ et $H$. Et, ces fonctions auxiliaires viennent d'être évaluées dans les Lemme 4.2, Corollaires 4.4 et 4.5. Donc, il n'y a qu'à appliquer ces informations aux $J_{1}$ et $J_{2}$. Le tableau suivant signifie, par exemple, que l'inégalité $\mathrm{du}$ cas (A) dans la proposition est une conséquence des $\left(12^{\circ}\right),\left(2^{\circ}\right),\left(10^{\circ}\right)$ et du Lemme 4.2:
$(\mathrm{A}) \leftarrow\left(12^{\circ}\right),\left(2^{\circ}\right),\left(10^{\circ}\right)$;
(B) $\leftarrow\left(11^{\circ}\right),\left(2^{\circ}\right),\left(6^{\circ}\right)$;
(C) $\leftarrow\left(8^{\circ}\right),\left(1^{\circ}\right),\left(9^{\circ}\right)$;
(D) $\leftarrow\left(7^{\circ}\right),\left(1^{\circ}\right),\left(5^{\circ}\right)$;
$(\mathrm{E}) \leftarrow\left(6^{\circ}\right),\left(3^{\circ}\right),\left(11^{\circ}\right)$;
$(\mathrm{F}) \longleftarrow\left(5^{\circ}\right),\left(3^{\circ}\right),\left(7^{\circ}\right)$.
C.Q.F.D.

5. Intégrales dépendantes aux paramètres (Suite). Dans ce paragraphe, nous allons évaluer les deux intégrales dépendantes aux paramètres.

Soit, premièrement, 


$$
\begin{aligned}
f(z) & =f\left(z ; L, a, b ; \alpha, \alpha^{\prime}, \beta, \beta^{\prime}\right) \\
= & z\{L(z+a)+1\}^{\alpha}\{L(z+b)+1\}^{\alpha^{\prime}}|z-a|^{-\beta}|z-b|^{-\beta^{\prime}} \\
& \times\left\{L^{2}|z-a|(|z-a|+|z-b|)+1\right\}^{(\beta-1) / 2} \\
& \times\left\{L^{2}|z-b|(|z-a|+|z-b|)+1\right\}^{\left(\beta^{\prime}-1\right) / 2} \\
& \times\left\{L(|z-a|+|z-b|)+(L|z-a|)^{\beta}+(L|z-b|)^{\beta^{\prime}}\right\} \\
& \times \exp \left[L^{2}\left\{(a-b)^{2}-(|z-a|+|z-b|)^{2}\right\}\right],
\end{aligned}
$$

où les $\left(L, a, b, \alpha, \alpha^{\prime}, \beta, \beta^{\prime}\right)$ sont des paramètres réels satisfaisant à

$$
\begin{aligned}
& L>0 ; \quad 0<a<1, \quad 0<b<1, \quad a+b=1 ; \\
& \alpha \geqq 0, \quad \alpha^{\prime} \geqq 0 ; \quad 0 \leqq \beta<1 \text { et } 0 \leqq \beta^{\prime}<1 .
\end{aligned}
$$

LEMmE 5.1. Sous l'hypothèse de (5.2), nous avons

$$
\int_{0}^{\infty} f\left(z ; L, a, b ; \alpha, \alpha^{\prime}, \beta, \beta^{\prime}\right) d z \leqq \text { Cte. } L^{\beta+\beta^{\prime}-2}(L+1)^{\alpha+\alpha^{\prime}+1},
$$

où la constante dépend aux $\left(\alpha, \alpha^{\prime}, \beta, \beta^{\prime}\right)$ mais elle est indépendante des $(L, a, b)$.

Preuve. Remarquons d'abord que la fonction $f$ est invariante par l'échange simultané des $(a, \alpha, \beta)$ et $\left(b, \alpha^{\prime}, \beta^{\prime}\right)$. On peut donc supposer que

$$
0<a \leqq 1 / 2 \leqq b<1 .
$$

On partage l'intégrale de (5.3) en quatre parties:

$$
\int_{0}^{\infty} f(z) d z=\int_{0}^{a}+\int_{a}^{b}+\int_{b}^{2}+\int_{2}^{\infty}=I_{1}+I_{2}+I_{3}+I_{4},
$$

on pose de plus $I_{5}=I_{1}+I_{2}+I_{3}$.

Majoration de $I_{4}$ : Si $z \geqq 2$, on a $f(z) \leqq$ Cte. $L^{\beta+\beta^{\prime-1}}(L z+1)^{\alpha+\alpha \prime} e^{-2(L z)^{2}}$, parce que $(|z-a|+|z-b|)^{2}-(a-b)^{2} \geqq 2 z^{2}$. On a donc

$$
I_{4} \leqq \text { Cte. } L^{\beta+\beta \prime-2} e^{-L^{2}} \text {. }
$$

Majoration de $I_{5}$ lorsque $L \leqq 1$ : Si $0<z \leqq 2$, on a

$$
\begin{gathered}
f(z) \leqq \text { Cte. }\left(L^{\beta^{\prime}}|z-a|^{-\beta}+L^{\beta}|z-b|^{-\beta^{\prime}}\right), \text { d'où } \\
I_{5} \leqq \text { Cte. }\left(L^{\beta}+L^{\beta^{\prime}}\right) .
\end{gathered}
$$

Par (5.6) et (5.7), nous avons (5.3) lorsque $0<L \leqq 1$.

Majoration de $I_{2}$ lorsque $L \geqq 1$ : Si $a=b$, alors $I_{2}=0$ par définition, supposons donc que $b>a$. Soit $a<z<b$ et posons $c=b-a, x=z-a$ et $y=b-z$. On voit que 


$$
f(z) \leqq \text { Cte. } L^{\alpha+\alpha^{\prime}+\beta+\beta^{\prime}-1} c^{-1}\left\{(c / x)^{(\beta+1) / 2}(c / y)^{\left(\beta^{\prime}+1\right) / 2}+(c / y)^{\left(\beta^{\prime}+1\right) / 2}+(c / x)^{(\beta+1) / 2}\right\} .
$$

On a donc

$$
I_{2} \leqq \text { Cte. } L^{\alpha+\alpha^{\prime}+\beta+\beta^{\prime}-1} .
$$

Majoration de $I_{3}$ lorsque $L \geqq 1$ : Soit $b<z \leqq 2$, et posons $c L=M$ et $w=(z-a)(z-b) L^{2}$. Alors,

$$
\begin{aligned}
L^{1-\alpha-\alpha^{\prime}-\beta-\beta^{\prime}} & I_{3} \leqq \text { Cte. } \int_{0}^{3 L^{2}} h(w) e^{-4 w} d w, \text { avec } \\
h(w)= & w^{-\beta^{\prime}}(w+1)^{\left(\beta^{\prime}-1\right) / 2}\left(w+M^{2}+1\right)^{(\beta-1) / 2}\left(w+M^{2}\right)^{\left(\beta^{\prime}-\beta-1\right) / 2} \\
& \times\left\{\sqrt{w+M^{2}}+\left(w+M^{2}\right)^{\beta / 2}+\left(\frac{w^{2}}{w+M^{2}}\right)^{\beta^{\prime} / 2}\right\} .
\end{aligned}
$$

On voit, sans difficulté, que $\int_{0}^{\infty} h(w) e^{-4 w} d w$ est majorée par une constante indépendante de $M>0$. Donc, on a

$$
I_{3} \leqq \text { Cte. } L^{\alpha+\alpha^{\prime}+\beta+\beta^{\prime}-1} \text {. }
$$

Majoration de $I_{1}$ lorsque $L \geqq 1$ : Posons également $w=(z-a)(z-b) L^{2}$. On a alors

$$
L^{1-\alpha-\alpha^{\prime}-\beta^{-\beta^{\prime}}} I_{1} \leqq \text { Cte. } \int_{0}^{a b L^{2}} h^{\prime}(w) e^{-4 w} d w
$$

où $h^{\prime}(w)$ est égale à $h(w)$ ci-dessus mais avec les $\beta$ et $\beta^{\prime}$ permutés. Donc, on a

$$
I_{1} \leqq \text { Cte. } L^{\alpha+\alpha^{\prime}+\beta+\beta^{\prime}-1} \text {. }
$$

Le Lemme 5.1 est maintenant établi.

C.Q.F.D.

La deuxième intégrale que nous considérons dans ce paragraphe est la suivante: Posons

$$
\begin{aligned}
g(z)= & g(z ; M ; \alpha, \beta, \gamma, \delta, \rho) \\
= & z|z-1|^{\alpha}(z+1)^{\beta}(M|z-1|+1)^{\gamma}\{M(z+1)+1\}^{\delta} \\
& \times\left\{M^{2}(z-1)^{2}+M(z+1)+1\right\}^{-\rho} \exp \left\{-M^{2}(z-1)^{2}\right\},
\end{aligned}
$$

où les $(M, \alpha, \beta, \gamma, \delta, \rho)$ sont des constantes satisfaisant à la suivante:

(5.12) $M>0 ; \alpha>-1, \alpha+\beta+2 \neq 0 ; \gamma \in \boldsymbol{R}, \delta \in \boldsymbol{R}$ et $\rho \in \boldsymbol{R}$.

LEMmE 5.2. Sous l'hypothèse de (5.12), nous avons

(5.13) $\int_{0}^{\infty} g(z ; M ; \alpha, \beta, \gamma, \delta, \rho) d z \leqq$ Cte. $\left(\frac{M+1}{M}\right)^{\operatorname{Max}(\alpha+\beta+2,0)}(M+1)^{\delta-\alpha-\rho-1}$,

où la constante dépend aux $(\alpha, \beta, \gamma, \delta, \rho)$ mais elle est indépendante de $M$. 
Preuve. Partageons l'intégrale de (5.13) en trois parties:

$$
\int_{0}^{\infty} g(z) d z=\int_{0}^{1 / 2}+\int_{1 / 2}^{2}+\int_{2}^{\infty}=J_{1}+J_{2}+J_{3},
$$

on pose de plus $J_{4}=J_{1}+J_{2}$.

Majoration de $J_{3}$ : $\mathrm{Si} z \geqq 2$, on a

$$
\begin{gathered}
M^{\alpha+\beta+1} g(z) \leqq \text { Cte. }(M z)^{\alpha+\beta+1} \times(M z+1)^{\gamma+\delta-2 \rho} \exp \left(-M^{2} z^{2} / 4\right), \quad \text { donc } \\
J_{3} \leqq \text { Cte. } M^{-\operatorname{Max}(\alpha+\beta+2,0)} \exp \left(-M^{2} / 8\right) .
\end{gathered}
$$

Majoration de $J_{4}$ lorsque $M \leqq 1$ : Si $0<z \leqq 2$, on a $g(z) \leqq$ Cte. $|z-1|^{\alpha}$, donc

$$
J_{4} \leqq \text { Cte. }
$$

Majoration de $J_{1}$ lorsque $M \geqq 1$ : Si $0<z \leqq 1 / 2$, on a

$$
\begin{aligned}
g(z) & \leqq \text { Cte. } M^{\gamma+\delta-2 \rho} \exp \left(-M^{2} / 4\right), \quad \text { donc } \\
J_{1} & \leqq \text { Cte. } M^{\gamma+\delta-2 \rho} \exp \left(-M^{2} / 4\right) .
\end{aligned}
$$

Majoration de $J_{2}$ lorsque $M \geqq 1$ : Si $1 / 2 \leqq z \leqq 2$, on a

$$
\begin{gathered}
g(z) \leqq \text { Cte. } M^{\delta-\alpha} v^{\alpha}(v+1)^{\gamma}\left(v^{2}+M\right)^{-\rho} \exp \left(-v^{2}\right) \\
\text { avec } v=M|v-1| . \text { Donc, } \\
J_{2} \leqq \text { Cte. } M^{\delta-\alpha-\rho-1} .
\end{gathered}
$$

Le Lemme 5.2 est maintenant établi.

C.Q.F.D.

6. Fonction auxiliaire $E(t, P, Q ; \lambda, \mu, \nu ; c)$. Soit $t>0$ et désignons par $P=(s, x), Q=\left(s^{\prime}, y\right), \quad R=\left(s^{\prime \prime}, z\right)$ etc. des points génériques de $\overline{R_{+}^{n}}=\left\{(s, x) ; s \in \boldsymbol{R}^{n-1}\right.$ et $\left.x \geqq 0\right\}$ et par $d R$ la mesure de Lebesgue $d s^{\prime \prime} d z$ dans $R^{n}$, où $n \geqq 2$. Définissons

$$
\begin{aligned}
& E(t, P, Q ; \lambda, \mu, \nu ; c)=t^{\lambda-(1 / 2)}\left(A_{P Q}+t\right)^{\mu-(1 / 2)} \\
& \quad \times\left(B_{P Q}+\left|s-s^{\prime}\right|+t+\sqrt{\left.t A_{P Q}\right)^{\nu+1-n}} \exp \left\{-c\left(t^{-1} B_{P Q}+\left|s-s^{\prime}\right|\right)\right\}\right. \\
& \text { avec } A_{P Q}=(\sqrt{x}+\sqrt{y})^{2} \text { et } B_{P Q}=(\sqrt{x}-\sqrt{y})^{2},
\end{aligned}
$$

où $c$ est une constante fixe positive et les $\lambda, \mu$ et $\nu$ sont des constantes réelles. Le but de ce paragraphe est de donner des inégalités de type de composition par rapport à $(t, P)$ des $E$ utilisées dans les $\S \S 2$ et 3 .

LEMME 6.1. Supposons que

$$
\begin{gathered}
-1<\lambda<-1 / 2, \quad-1<\lambda^{\prime}<-1 / 2 ; \quad \mu \geqq 1 / 2, \quad \mu^{\prime} \geqq 1 / 2 ; \\
\nu>0, \quad \nu^{\prime}>0 \text { et } \nu+\nu^{\prime}<n-1 .
\end{gathered}
$$

Alors, nous avons l'inégalité suivante: 


$$
\begin{aligned}
& \int_{0}^{t} d t^{\prime} \int_{R_{+}^{n}} E\left(t-t^{\prime}, P, R ; \lambda, \mu, \nu ; c\right) E\left(t^{\prime}, R, Q ; \lambda^{\prime}, \mu^{\prime}, \nu^{\prime} ; c\right) d R \\
& \quad \leqq K \cdot E\left(t, P, Q ; \lambda+\lambda^{\prime}+1, \mu+\mu^{\prime}, \nu+\nu^{\prime} ; c\right),
\end{aligned}
$$

où $K$ est une constante positive dépendante $\grave{a}\left(\lambda, \mu, \nu, \lambda^{\prime}, \mu^{\prime}, \nu^{\prime}, c\right)$ mais indépendante des $(t, P, Q)$.

Démonstration: Nous effectuons les intégrations d'abord par rapport à $s^{\prime \prime}$, ensuite à $t^{\prime}$ et finalement à $z$. Premièrement, nous savons le fait suivant: Soient $\left(\nu, \nu^{\prime}\right)$ satisfaisant à (6.2), alors

$$
\begin{aligned}
& \left.\int_{\boldsymbol{R}^{n-1}}\left(\left|s-s^{\prime \prime}\right|+M\right)^{\nu+1-n}\left(\left|s^{\prime \prime}-s^{\prime}\right|+N\right)^{\nu \nu^{\prime}+1-n} e^{-c\left(\left|s-s^{\prime \prime}\right|+\left|s^{\prime \prime}-s^{\prime}\right|\right.}\right) d s^{\prime \prime} \\
& \quad \leqq K\left(\left|s-s^{\prime}\right|+M+N\right)^{\nu+\nu^{\prime}+1-n} e^{-c\left|s-s^{\prime}\right|}, \text { quels que soient } s, s^{\prime} \in \boldsymbol{R}^{n-1},
\end{aligned}
$$

où $K>0$ est indépendante des $\left(M, N, s, s^{\prime}\right)$ (Vérification facile). Nous l'appliquons pour $M=B_{P R}+t-t^{\prime}+\sqrt{\left(t-t^{\prime}\right) A_{P R}}$ et $N=B_{R Q}+t^{\prime}+\sqrt{t^{\prime} A_{R Q}}$, pour $0<t^{\prime}<t$, et remarquons que $4(M+N) \geqq B_{P Q}+t+\sqrt{t A_{P Q}}$. Alors, grâce à l'hypothèse (6.2) sur $\left(\mu, \mu^{\prime}\right)$, nous avons

$$
\begin{aligned}
& \left(B_{P Q}+\left|s-s^{\prime}\right|+t+\sqrt{t A_{P Q}}\right)^{n-1-\nu-\nu^{\prime}} e^{c\left|s-s^{\prime}\right|} \int_{R^{n-1}} E(\cdots) E(\cdots) d s^{\prime \prime} \\
& \leqq \text { Cte. }\left(A_{P R}+t\right)^{\mu-(1 / 2)}\left(A_{R Q}+t\right)^{\mu^{\prime}-(1 / 2)} F\left(t, t^{\prime}, x, y, z\right), \quad \text { où } \\
& F\left(t, t^{\prime}, x, y, z\right)=\left(t-t^{\prime}\right)^{\lambda-(1 / 2)} t^{\prime \lambda^{\prime}-(1 / 2)} \exp \left\{-c\left(\frac{B_{P R}}{t-t^{\prime}}\right)+\left(\frac{B_{R Q}}{t^{\prime}}\right)\right\}
\end{aligned}
$$

Pour intégrer par rapport à $t^{\prime}$, nous appliquons la Proposition 4.1, (A) avec $\alpha=\lambda-(1 / 2), \beta=\lambda^{\prime}-(1 / 2), \rho=0, A=B_{P R} / t, B=B_{R Q} / t$ et $P=1$. Alors,

$$
\begin{aligned}
& \int_{0}^{t} F\left(t, t^{\prime}, x, y, z\right) d t^{\prime} \leqq \text { Cte. } t^{\lambda+\lambda^{\prime}+(1 / 2)} B_{P R}^{\lambda+(1 / 2)} B_{R Q}^{\lambda^{\prime}+(1 / 2)} \\
& \times\left\{\sqrt{B_{P R}}\left(\sqrt{B_{P R}}+\sqrt{B_{R Q}}\right)+t\right\}^{-\lambda-1}\left\{\sqrt{B_{R Q}}\left(\sqrt{B_{P R}}+\sqrt{B_{R Q}}\right)+t\right\}^{-\lambda^{\prime}-1} \\
& \quad \times\left\{\sqrt{B_{P R}}+\sqrt{B_{R Q}}+\sqrt{t}\left(B_{P R} / t\right)^{-\lambda-(1 / 2)}+\sqrt{t}\left(B_{R Q} / t\right)^{-\lambda^{\prime}-(1 / 2)}\right\} \\
& \quad \times \exp \left\{-c\left(\sqrt{B_{P R}}+\sqrt{B_{R Q}}\right)^{2} / t\right\} .
\end{aligned}
$$

Dernièrement pour intégrer par rapport à $z$, nous faisons appel à l'aide du Lemme 5.1 avec $\alpha=2 \mu-1, \alpha^{\prime}=2 \mu^{\prime}-1, \beta=-2 \lambda-1, \beta^{\prime}=-2 \lambda^{\prime}-1$, $L=\sqrt{c A_{P Q} / t}, L a=\sqrt{c x / t}$ et $L b=\sqrt{c y / t}$. Alors, nous avons

$$
\begin{aligned}
& \int_{0}^{\infty}\left(A_{P R}+t\right)^{\mu-(1 / 2)}\left(A_{R Q}+t\right)^{\mu^{\prime}-(1 / 2)} d z \int_{0}^{t} F\left(t, t^{\prime}, x, y, z\right) d t^{\prime} \\
& \quad \leqq \text { Cte. } t^{\lambda+\lambda \lambda^{\prime+(1 / 2)}}\left(A_{P Q}+t\right)^{\mu+\mu^{\prime}-(1 / 2)} \exp \left(-c B_{P Q} / t\right),
\end{aligned}
$$

ce qui démontre (6.3).

C.Q.F.D. 
Nous pouvons poser $P=Q$ dans (6.3):

$$
\begin{aligned}
& \int_{0}^{t} d t^{\prime} \int_{R^{n-1}} E\left(t-t^{\prime}, P, R ; \lambda, \mu, \nu ; c\right) E\left(t^{\prime}, R, P ; \lambda^{\prime}, \mu^{\prime}, \nu^{\prime} ; c\right) d R \\
& \quad \leqq K t^{\lambda+\lambda^{\prime}+1}(x+t)^{\mu+\mu^{\prime}}\{t(x+t)\}^{\left(\nu+\nu^{\prime}-n\right) / 2},
\end{aligned}
$$

si (6.2) a lieu.

LEMME 6.2. L'inégalité (6.5) reste vraie sous l'hypothèse suivante au lieu de (6.2):

$$
\begin{aligned}
& -1<\lambda^{\prime}<-1 / 2<\lambda, \quad \mu<1 / 2 \leqq \mu^{\prime}, \\
& \lambda+\mu<0, \quad \lambda^{\prime}+\mu^{\prime}<0, \quad \lambda+\lambda^{\prime}+\mu+\mu^{\prime}+1>0, \\
& \nu>0, \quad \nu^{\prime}>0 \text { et } \nu+\nu^{\prime}<n-1 .
\end{aligned}
$$

PreUve. Analoguement à (6.4), nous avons d'abord

$$
\begin{aligned}
& \{t(x+t)\}^{\left(n-1-\nu-\nu^{\prime}\right) / 2} \int_{R^{n-1}} E\left(t-t^{\prime}, P, R ; \cdots\right) E\left(t^{\prime}, R, P ; \cdots\right) d s^{\prime \prime} \\
& \quad \leqq \text { Cte. } G\left(t, t^{\prime}, x, z\right), \text { où } \\
& G\left(t, t^{\prime}, x, z\right)=\left(t-t^{\prime}\right)^{\lambda-(1 / 2)} t^{\prime \lambda^{\prime}-(1 / 2)}\left(A_{P R}+t-t^{\prime}\right)^{\mu-(1 / 2)} \\
& \quad \times\left\{A_{P R}^{\mu^{\prime}-(1 / 2)}+t^{\prime \mu^{\prime}-(1 / 2)}\right\} \exp \left\{-c B_{P R}\left(\frac{1}{t-t^{\prime}}+\frac{1}{t^{\prime}}\right)\right\} \cdot
\end{aligned}
$$

Nous utilisons la Proposition 4.1, (C) avec $0<A=B=c B_{P R} / t<P=c A_{P R} / t$, $\rho=(1 / 2)-\mu, \alpha=\lambda-(1 / 2)$, et $\beta=\lambda^{\prime}+\mu^{\prime}-1$ ou $\beta=\lambda^{\prime}-(1 / 2)$. Alors,

$$
\begin{aligned}
& \int_{0}^{t} G\left(t, t^{\prime}, x, z\right) d t^{\prime} \leqq \text { Cte. } t^{\lambda+\lambda^{\prime}+(1 / 2)} B_{P R}^{\lambda^{\prime}+(1 / 2)} A_{P R}^{\lambda+\mu+\mu^{\prime}-(1 / 2)} \\
& \quad \times\left(A_{P R}+t\right)^{-\lambda-(1 / 2)}\left(B_{P R}+t\right)^{-\lambda^{\prime}-1} \exp \left(-4 c B_{P R} / t\right)
\end{aligned}
$$

Dernièrement pour intégrer par rapport à $z$, nous utilisons le Lemme 5.2 avec $M=2 \sqrt{c x / t}, \quad \alpha=2 \lambda^{\prime}+1, \quad \beta=2\left(\lambda+\mu+\mu^{\prime}\right)-1, \quad \gamma=-2 \lambda^{\prime}-2$, $\delta=-2 \lambda-1$ et $\rho=0$. Alors,

$$
\int_{0}^{\infty} d z \int_{0}^{t} G\left(t, t^{\prime}, x, z\right) d t^{\prime} \leqq \text { Cte. } t^{\lambda+\lambda^{\prime}+(1 / 2)}(x+t)^{\mu+\mu^{\prime}-(1 / 2)} .
$$

Celle-ci n'est autre que (6.5).

C.Q.F.D.

7. Préparation du calcul du trace. Soient $t>0, \hat{s} \in R^{n-1}(n \geqq 2)$ et $x \geqq 0$. Définissons

$$
\begin{aligned}
& \hat{\gamma}(t ; \hat{s}, x)=\frac{\sigma}{\sinh (\sigma t)} I_{0}\left(\frac{2 \sigma x}{\sinh (\sigma t)}\right) \exp \{-2 \sigma x \operatorname{coth}(\sigma t)\}, \\
& \text { avec } \sigma=\left\{\sum_{\alpha=1}^{n-1} \hat{s}_{\alpha}^{2}+1\right\}^{1 / 2} ;
\end{aligned}
$$




$$
\kappa(t)=\int_{0}^{\infty} \gamma(t ; x) d x, \quad \text { où } \gamma(t ; x)=(2 \pi)^{1-n} \int_{R^{n-1}} \hat{\gamma}(t ; \hat{s}, x) d \hat{s} \text {. }
$$

Dans ce paragraphe, nous allons obtenir le développement asymptotique de $\kappa(t)$ lorsque $t \downarrow 0$. En particulier pour $n=2$, nous allons considérer également le comportement de la fonction

$$
\kappa_{(0)}(t ; \delta)=\int_{0}^{\delta / 2} \gamma(t ; x) d x,
$$

où $\delta$ est un paramètre positif. Il s'agira du cas où $\delta$ est petit et $t$ tend vers zéro. Commençons par $\kappa(t)$.

Proposition 7.1. La fonction $\kappa(t)$ admet le développement suivant sur le segment $0<t \leqq 1$ :

$$
t^{n-1} \kappa(t)= \begin{cases}\psi_{0}(t) & , \text { si } n=\text { impair } \geqq 3, \\ \psi_{1}(t)+t^{n-2} \psi_{2}(t) \log \frac{1}{t} & , \text { si } n=\text { pair } \geqq 4, \\ \frac{1}{2 \pi} \log \frac{1}{t}+\psi_{3}(t)+t^{2} \psi_{4}(t) \log \frac{1}{t}, & \text { si } n=2,\end{cases}
$$

où les $\psi_{0}(t), \cdots, \psi_{4}(t)$ sont des fonctions holomorphes de $t \grave{a} t=0$.

Si $n \geqq 3$, nous avons

$$
\begin{aligned}
& \left\{t^{n-1} \kappa(t)\right\}_{t=0}=(n-1) k_{n} \int_{0}^{\infty}\left[\frac{\xi+1}{2}\right] \xi^{-n} d \xi, \\
& \text { avec } k_{n}=(n-1)(2 \pi)^{1-n} \omega_{n-1} \text { et } \omega_{n-1}=\pi^{(n-1) / 2} \Gamma\left(\frac{n+1}{2}\right)^{-1},
\end{aligned}
$$

où [z] désigne la partie entière de $z$.

Démonstration. Dans la définition de $\kappa(t)$, nous échangeons l'ordre d'intégrations par rapport à $\hat{s}$ et $x$. D'après la formule de Lipschitz

nous avons

$$
\int_{0}^{\infty} I_{0}(y) e^{-y \cosh A} d y=1 / \sinh A, \text { pour } A>0,
$$

$$
\kappa(t)=(2 \pi)^{1-n} \int_{R^{n-1}} 2 \frac{d \hat{s}}{\sinh (\sigma t)} .
$$

Passons aux coordonnées polaires:

$$
\rho=\left\{\sum_{\alpha=1}^{n-1} \hat{s}_{\alpha}^{2}\right\}^{1 / 2}, \quad \zeta_{\alpha}=\hat{s}_{\alpha} / \rho, \quad 1 \leqq \alpha \leqq n-1 .
$$

Alors, $d \hat{\boldsymbol{s}}=\rho^{n-2} d \rho d S_{\zeta}$ et $\int_{\rho=1} d S_{\zeta}=(n-1) \omega_{n-1}$. Donc, $\kappa(t)$ est représentée par une intégrale unie-dimensionnelle: 


$$
\kappa(t)=k_{n} \int_{0}^{\infty} \frac{\rho^{n-2} d \rho}{2 \sinh (\sigma t)} .
$$

Posons de plus $r=\sigma t=t \sqrt{\rho^{2}+1}$. Nous avons finalement

$$
\frac{t^{n-1}}{k_{n}} \kappa(t)=\int_{t}^{\infty} \lambda(r)\left(r^{2}-t^{2}\right)^{(n-3) / 2} d r, \quad \text { où } \quad \lambda(r)=\frac{r}{2 \sinh r} .
$$

$\lambda(r)$ est une fonction holomorphe de $r^{2}$ dans $|r|<\pi / 2$ et $0 \leqq r<\infty$ et de décroissance rapide à $r \rightarrow \infty$.

Soit d'abord $n=i m p a i r \geqq 3$. Alors, l'intégrand de (7.6) est holomorphe par rapport à $(r, t)$, d'où (7.4).

Soit ensuite $n=$ pair $\geqq 4$. Posons $n=2 p+4(p=0,1, \cdots)$. Alors, le membre à droite de (7.6) devient

$$
\int_{t}^{2} \lambda(r)\left(r^{2}-t^{2}\right)^{p+(1 / 2)} d r+\int_{2}^{\infty} \lambda(r)\left(r^{2}-t^{2}\right)^{p+(1 / 2)} d r
$$

Le dernier terme est évidemment holomorphe sur $0 \leqq t \leqq 1$. Quant au premier terme, il est la série convergente des termes

$$
\int_{t}^{2} r^{2 j}\left(r^{2}-t^{2}\right)^{p+(1 / 2)} d r=t^{2 j+2 p} \int_{0}^{\theta(t)}(\cosh \theta)^{2 j}(\sinh \theta)^{2 p} d \theta
$$

avec $j=0,1,2, \cdots$ et $\theta(t)=$ arc. $\cosh (2 / t)$, d'où l'on a (7.4).

Soit dernièrement $n=2$. Alors, $k_{2}=1 / \pi$ et le membre à droite de (7.6) est

$$
\frac{1}{2} \int_{t}^{2} \frac{d r}{\sqrt{r^{2}-t^{2}}}+\int_{t}^{2} \frac{\lambda(r)-\lambda(0)}{\sqrt{r^{2}-t^{2}}} d r+\int_{2}^{\infty} \frac{\lambda(r) d r}{\sqrt{r^{2}-t^{2}}} .
$$

Le premier est égal à $(1 / 2) \operatorname{arc} \cdot \cosh (2 / t)=(1 / 2) \log \left\{\left(2+\sqrt{4-t^{2}}\right) / t\right\}$. Pour les autres termes, le raisonnement est analogue à celui aux cas précédents.

Si $n \geqq 3$, il existe la valeur de $t^{n-1} \kappa(t)$ à $t=0$ et elle se donne

$$
\begin{aligned}
k_{n}^{-1}\left\{t^{n-1} \kappa(t)\right\}_{t=0} & =\int_{0}^{\infty} \lambda(r) r^{n-3} d r=(n-2) ! \sum_{j=0}^{\infty}(2 j+1)^{1-n} \\
& =(n-1) ! \int_{0}^{\infty}\left[\frac{\xi+1}{2}\right] \xi^{-n} d \xi
\end{aligned}
$$

Maintenant, soit $n=2$ et considérons la fonction $\kappa_{(0)}(t ; \delta)$ définie par (7.3). Nous allons démontrer la suivante

Proposition 7.2. Fixons un $\delta>0$. Alors, lorsque $t \downarrow 0$, nous avons une formule asymptotique 


$$
\kappa_{(0)}(t ; \delta)=\frac{1}{4 \pi t} \log \frac{1}{t}+\frac{A}{t}+O(1 / \sqrt{t}), \text { pour } n=2,
$$

où $A$ est une constante dépendante à $\delta$.

$\delta$ est fixe dans la proposition, mais dans la démonstration, nous le regardons comme une variable parcourant un intervalle $\left(0, \delta_{0}\right]$, parce que nous aurons besoin du comportement à $\delta \rightarrow 0$ dans la proposition suivante. Posons d'abord

$$
\kappa_{(\infty)}(t ; \delta)=\kappa(t)-\kappa_{(0)}(t ; \delta)=\int_{\delta / 2}^{\infty} \gamma(t ; x) d x .
$$

Grâce à (7.4), il suffit de vérifier la formule asymptotique

$$
\kappa_{(\infty)}(t ; \delta)=\frac{1}{4 \pi t} \log \frac{1}{t}+\frac{B}{t}+O(1 / \sqrt{t}), \quad B=\text { Cte. }(\delta),
$$

lorsque $t \downarrow 0$. Pour commencer, nous utilisons une représentation intégrale

$$
I_{0}(z)=\frac{1}{\pi} \int_{-1}^{1} e^{z \zeta}\left(1-\zeta^{2}\right)^{-1 / 2} d \zeta,
$$

et effectuons l'intégration par rapport à $x$. Alors, nous avons

$$
\begin{aligned}
2 \pi^{2} t \kappa_{(\infty)}(t ; \delta)= & \int_{-1}^{1} \frac{d \zeta}{\sqrt{1-\zeta^{2}}} \int_{t}^{\infty}(\operatorname{ch} r-\zeta)^{-1} \\
& \times \exp \left\{-\frac{r \delta}{t}\left(\operatorname{ch} r-\frac{\zeta}{\operatorname{sh} r}\right)\right\} \frac{r d r}{\sqrt{r^{2}-t^{2}}} .
\end{aligned}
$$

Soit maintenant $0<t \ll 1 / 2$, et partageons le membre à droite en trois parties:

$$
\begin{aligned}
2 \pi^{2} t \kappa_{(\infty)}(t ; \delta) & =\int_{1 / 2}^{1} d \zeta \int_{t}^{1} d r+\int_{-1}^{1 / 2} d \zeta \int_{t}^{1} d r+\int_{-1}^{1} d \zeta \int_{1}^{\infty} d r \\
& \equiv f_{1}(t)+f_{2}(t)+f_{3}(t) .
\end{aligned}
$$

On voit, sans difficulté, que

$$
0<f_{2}(t)+f_{3}(t) \leqq K \exp (-a \delta / t), \quad K>0 \text { et } a>0,
$$

où $K$ et $a$ sont des constantes indépendantes de $(t, \delta)$. Il nous reste à évaluer $f_{1}(t)$ qui est plus difficile. Posons

$$
\begin{aligned}
& X=X(r, t)=\frac{r / t}{\operatorname{sh} r}(\operatorname{ch} r-1), \quad Y=Y(r, t)=\frac{r / t}{2 \operatorname{sh} r}, \\
& \text { et } J(X, Y ; \delta)=\int_{0}^{Y} \frac{e^{-\delta \zeta}}{(\zeta+X) \sqrt{\zeta}}\left(1-\frac{\zeta}{4 Y}\right)^{-1 / 2} d \zeta .
\end{aligned}
$$

On a alors 


$$
f_{1}(t)=\int_{t}^{1} e^{-\delta X} \sqrt{Y} J(X, Y ; \delta) \frac{r d r}{\sqrt{r^{2}-t^{2}}} .
$$

Lorsque $r$ varie de $t$ à $1, X$ varie de $X_{0}(t)$ à $X_{1}(t)$, où

$$
X_{0}(t)=\frac{\operatorname{ch} t-1}{\operatorname{sh} t} \text { et } X_{1}(t)=\frac{\operatorname{ch} 1-1}{t \operatorname{sh} 1},
$$

et $Y$ est uniformément d'ordre $1 / t$, on peut done supposer que $t$ soit si petit que $Y$ soit toujours plus grand qu'l. Posons

(7.16) $J(X ; \delta)=J(X,+\infty ; \delta)$ et $J^{\prime}(X, Y ; \delta)=J(X, Y ; \delta)-J(X ; \delta)$.

Lemme 7.1. Soient $X>0, Y \geqq 1$ et $\delta>0$. Alors,

$$
\begin{gathered}
\left(1^{\circ}\right) \quad J(X ; \delta)=\sqrt{\pi / X} \Psi(1 / 2,1 / 2 ; \delta X)=O(1 / \sqrt{X(\delta X+1)}), \\
\left(2^{\circ}\right) \quad J^{\prime}(X, Y ; \delta)=O(1 / \sqrt{Y(\delta Y+1)}),
\end{gathered}
$$

où les $O$ sont uniformes par rapport à $(X, Y, \delta)$.

Preuve. Pour $\left(1^{\circ}\right)$, voir [3] Chap. 6 du Vol. 1 et (4.13). Pour $\left(2^{\circ}\right)$, $J^{\prime}(X, Y ; \delta)$ est égale à

$$
\int_{0}^{Y} \frac{e^{-\delta \zeta}}{(\zeta+X) \sqrt{\zeta}}\left\{\left(1-\frac{\zeta}{4 Y}\right)^{-1 / 2}-1\right\} d \zeta-\int_{Y}^{\infty} \frac{e^{-\delta \zeta} d \zeta}{(\zeta+X) \sqrt{\zeta}}
$$

dont chaque intégrale est $O(1 / \sqrt{Y(\delta Y+1)})$.

C.Q.F.D.

Nous posons

$$
f_{4}(t)=\int_{t}^{1} e^{-\delta X} \sqrt{Y} J(X ; \delta) \frac{r d r}{\sqrt{r^{2}-t^{2}}},
$$

alors, le Lemme 7.1 entraîne tout de suite le suivant

LEMME 7.2. Nous avons

$$
\left|f_{1}(t)-f_{4}(t)\right| \leqq K \sqrt{t} / \sqrt{\delta+t},
$$

où $K$ est une constante indépendante de $(t, \delta)$.

Nous avons vu jusqu'ici que

$$
\left|2 \pi^{2} t \kappa_{(\infty)}(t ; \delta)-f_{4}(t)\right| \leqq K^{\prime} \sqrt{t} / \sqrt{\delta+t},
$$

avec une $K^{\prime}>0$ indépendante de $(t, \delta)$. Pour évaluer maintenant $f_{4}(t)$, nous allons la transformer en une intégrale par rapport à $X$. Posons

(7.22) $z=r^{2}$ et $\xi=t X(r, t)=\phi(z)$, donc $\phi(z)=r(\operatorname{ch} r-1) / \operatorname{sh} r$.

$\phi(z)$ est holomorphe en $z$ sur la demie-droite $z \geqq 0$, positive si $z>0$, $\phi^{\prime}(z)>0>\phi^{\prime \prime}(z)$ si $z>0, \phi(0)=0, \phi^{\prime}(0)=1 / 2$ et $\phi^{\prime \prime}(0)=-1 / 12$. On peut donc résoudre l'équation $\xi=\phi(z)$ par rapport à $z$ : 


$$
z=\psi(\xi), \text { avec } \psi^{\prime}(0)=2 \text {. }
$$

Posons encore

$$
\begin{aligned}
& \eta(\xi)=\sqrt{2 t Y}=\sqrt{r / \operatorname{sh} r}, \quad \psi_{1}(\xi)=\psi(\xi) / \xi \\
& \text { et } \eta_{1}(\xi)=\eta(\xi) \psi^{\prime}(\xi) .
\end{aligned}
$$

Ces fonctions sont holomorphes et positives. Les $\eta_{1}(\xi)$ et $1 / \psi_{1}(\xi)$ sont bornées lorsque $\xi$ varie de 0 à $\phi(1)$ et de plus $2 r d r=t \psi^{\prime}(\xi) d X$. Cela posé, nous avons

$$
\begin{aligned}
2 \sqrt{2} f_{4}(t) & =\int_{X_{0}(t)}^{X_{1}(t)} e^{-\delta X} \eta_{1}(\xi) J(X ; \delta) \frac{d X}{\sqrt{X \psi_{1}(\xi)-t}}=\int_{X_{0}(t)}^{1}+\int_{1}^{X_{1}(t)} \\
& \equiv f_{5}(t)+f_{6}(t) .
\end{aligned}
$$

Nous traitons d'abord $f_{5}(t)$. Posons $\xi_{0}=t X_{0}(t)$. Alors la fonction

$$
\psi_{2}\left(\xi, \xi_{0}\right)=\left\{\psi(\xi)-\psi\left(\xi_{0}\right)\right\} /\left(\xi-\xi_{0}\right)
$$

est holomorphe par rapport à $\left(\xi, \xi_{0}\right)$ et $\psi_{2}(0,0)=2$. Comme $t^{2}=\psi\left(\xi_{0}\right)$, nous avons par (7.25), $X \psi_{1}(\xi)-t=\left\{X-X_{0}(t)\right\} \psi_{2}\left(\xi, \xi_{0}\right)$. Donc,

$$
\begin{aligned}
& f_{5}(t)=\int_{X_{0}(t)}^{1} \eta_{2}(t, X) e^{-\delta X} J(X ; \delta) \frac{d X}{\sqrt{X-X_{0}(t)}}, \text { avec } \\
& \eta_{2}(t, X)=\eta_{1}(\xi) / \sqrt{\psi_{2}\left(\xi, \xi_{0}\right)} \text { (en particulier } \eta_{2}(0,0)=\sqrt{2} \text { ). }
\end{aligned}
$$

Partageons l'intégrale de (7.26) en deux parties:

$$
\begin{aligned}
f_{5}(t)= & \sqrt{2} \int_{X_{0}(t)}^{1} \frac{e^{-\delta X}}{\sqrt{X-X_{0}(t)}} J(X ; \delta) d X \\
& +\int_{X_{0}(t)}^{1}\left\{\eta_{2}(t, X)-\sqrt{2}\right\} \frac{e^{-\delta X}}{\sqrt{X-X_{0}(t)}} J(X ; \delta) d X \\
\equiv & f_{7}(t)+f_{8}(t) .
\end{aligned}
$$

Lemme 7.3. Lorsque $0<t \ll 1 / 2$, nous avons

$$
f_{8}(t)=O\left(t \log \frac{1}{t}\right)
$$

uniformément par rapport $\grave{a} \delta$. 7.1,

Preuve. $\quad \eta_{2}(t, X)-\sqrt{2}=O(t)$ si $0<X \leqq 1$ et donc par le Lemme

$$
\left|f_{8}(t)\right| \leqq \text { Cte. } t \int_{X_{0}(t)}^{1} \frac{d X}{\sqrt{X\left(X-X_{0}(t)\right)}} .
$$

Mais on sait que 


$$
\int_{X_{0}}^{1} \frac{d X}{\sqrt{X\left(X-X_{0}\right)}}=\log \frac{2-X_{0}+2 \sqrt{1-X_{0}}}{X_{0}}
$$

et que $X_{0}(t)=O(t)$ par (7.15), d'où (7.28).

C.Q.F.D.

LEMM 7.4. Lorsque $0<t \ll 1 / 2$, nous avons

$$
\frac{1}{\sqrt{2}} f_{7}(t)=\pi \log \frac{1}{t}+g_{7}(t)+\sqrt{\delta} h_{7}(t, \delta),
$$

où $g_{7}(t)\left(\right.$ resp. $\left.h_{7}(t, \delta)\right)$ est une fonction holomorphe de $t$ (resp. de $\left.(t, \delta)\right)$ $\grave{a} t=0($ resp. $\grave{a}(t, \delta)=(0,0))$.

Preuve. On sait ([3], Chap. 6 du Vol. 1, p. 266) que

$$
\begin{aligned}
e^{-\delta X} J(X ; \delta) & =\frac{\pi}{\sqrt{X}}+\sqrt{\delta} \Phi(\delta X), \\
\text { avec } \Phi(X) & =-2 \sqrt{\pi_{1}} F_{1}(1 / 2 ; 3 / 2 ;-X)
\end{aligned}
$$

où $\Phi(X)$ est une fonction entière de $X$. Donc,

$$
\int_{X_{0}(t)}^{1} \frac{\Phi(\delta X) d X}{\sqrt{X-X_{0}(t)}}=h_{7}(t, \delta)
$$

est holomorphe à $(t, \delta)=(0,0)$. D'autre part, le membre à droite de (7.29) (avec $X_{0}=X_{0}(t)$ ) est égal à $\log (1 / t)+g_{7}(t)$ avec une $g_{7}(t)$ holomorphe à $t=0$, d'où (7.30).

C.Q.F.D.

La propriété de la fonction $f_{5}(t)$ étant éclaircie, il faut maintenant regarder $f_{6}(t)$ dans (7.25). Notons que $\eta_{1}(0)=\psi_{1}(0)=2$, donc $f_{6}(t)$ s'écrit

$$
\begin{aligned}
f_{6}(t)= & \sqrt{2} \int_{1}^{x_{1}(t)} e^{-\delta X} J(X ; \delta) \frac{d X}{\sqrt{X}} \\
& +\int_{1}^{x_{1}(t)} e^{-\delta X} J(X ; \delta)\left\{\frac{\eta_{1}(\xi)}{\sqrt{X \psi_{1}(\xi)-t}}-\sqrt{\frac{2}{X}}\right\} d X \\
\equiv & f_{9}(t)+f_{10}(t) .
\end{aligned}
$$

LEMME 7.5. Nous avons

$$
\left|f_{10}(t)\right| \leqq K^{\prime \prime} t /(\delta+t),
$$

où $K^{\prime \prime}$ est une constante positive indépendante de $(t, \delta)$.

Preuve. Si $X \geqq 1$, on a

$$
\left|\eta_{1}(\xi) / \sqrt{X \psi_{1}(\xi)-t}-\sqrt{2 / X}\right| \leqq \text { Cte. } t X,
$$

donc, par le Lemme 7.1, $\left(1^{\circ}\right)$,

$$
\left|f_{10}(t)\right| \leqq \text { Cte. } t \int_{1}^{x_{1}(t)} \frac{e^{-\delta X}}{\sqrt{\delta X+1}} d X \leqq \text { Cte. } t /(\delta+t) . \quad \text { C.Q.F.D. }
$$


Dernièrement, $f_{9}(t)$ s'écrit

$$
\frac{1}{\sqrt{2 \pi}} f_{9}(t)=\int_{\delta}^{\xi_{1} \delta / t} e^{-y} \Psi(1 / 2,1 / 2 ; y) \frac{d y}{y},
$$

où $\xi_{1}=t X_{1}(t)=(\operatorname{ch} 1-1) / \operatorname{sh} 1$, donc $0<\xi_{1}<1 / 2$.

LEMmE 7.6. Soient $0<t \ll \xi_{1}$ et $\delta>0$. Alors,

$$
S i \xi_{1} \delta \leqq t, \quad f_{9}(t)=\sqrt{2} \pi \log \left(\xi_{1} / t\right)+O\left(\sqrt{\left.\xi_{1} \delta / t\right)} ;\right.
$$

$$
S i \xi_{1} \delta \geqq t, \quad 0<f_{9}(0)-f_{9}(t)=O\left((t / \delta)^{3 / 2} \exp \left(-\xi_{1} \delta / t\right)\right),
$$

uniformément par rapport $\grave{a}(t, \delta)$, où

$$
f_{9}(0)=\sqrt{2 \pi} \int_{\delta}^{\infty} e^{-y} \Psi(1 / 2,1 / 2 ; y) \frac{d y}{y} .
$$

Preuve. Il suffit seulement de remarquer que

$$
\Psi(1 / 2,1 / 2 ; y)=\left\{\begin{array}{l}
\sqrt{\pi}+O(\sqrt{y}), \quad \text { pour } y \downarrow 0, \\
O(1 / \sqrt{y}), \quad \text { pour } y \uparrow \infty .
\end{array}\right.
$$

Nous résumons le raisonnement sur l'estimation de $\kappa_{(\infty)}(t ; \delta)$. Nous pouvons écrire

$$
2 \pi^{2} t \kappa_{(\infty)}(t ; \delta)=\frac{1}{2 \sqrt{2}}\left\{f_{7}(t)+f_{9}(t)\right\}+f_{11}(t),
$$

où $f_{11}(t)=\left\{2 \pi^{2} t \kappa_{(\infty)}(t ; \delta)-f_{4}(t)\right\}+\left\{f_{8}(t)+f_{10}(t)\right\} / 2 \sqrt{2} . \quad$ D'après

Lemme 7.3 et Lemme 7.5 , on a

$$
\left|f_{11}(t)\right| \leqq K\left(\sqrt{\frac{t}{\delta+t}}+t \log \frac{1}{t}\right) .
$$

Fin de la démonstration de la Proposition 7.2: Fixons un $\delta>0$. Alors, lorsque $t \downarrow 0$, on a $f_{11}(t)=O(\sqrt{t})$ par (7.39),

$$
\begin{gathered}
\frac{1}{2 \sqrt{2}} f_{7}(t)=\frac{\pi}{2} \log \frac{1}{t}+\text { Cte. }+O(t) \text { par le Lemme } 7.4 \text {, et } \\
\frac{1}{2 \sqrt{2}} f_{9}(t)=\text { Cte. }+O(t \sqrt{t}) \text { par le Lemme } 7.6 \text {. }
\end{gathered}
$$

Nous avons ainsi vérifié la formule (7.9) ce qui finit la démonstration.

C.Q.F.D.

Proposition 7.3. Soit $n=2$. Nous nous donnons une fonction $a(x)$ de classe $C^{1}$ sur $0 \leqq x<\infty$ telle que $a(0)=0$. Alors, pour un $\delta>0$ fixe, nous avons une formule asymptotique suivante: 


$$
\int_{0}^{\delta} \gamma(t ; x) a(x) d x=\frac{C}{t}+O(1 / \sqrt{t}), \quad \text { lorsque } t \downarrow 0,
$$

oú $C$ est une constante indépendante de $t$.

Démonstration: $a^{\prime}(x)=d a / d x$ est continue et bornée. Et d'autre part $\gamma(t ; x)=-\left(\frac{\partial}{\partial x} \kappa_{(\infty)}\right)(t ; 2 x)$. Donc, nous avons

$$
\int_{0}^{\delta} \gamma(t ; x) a(x) d x=-\kappa_{(\infty)}(t ; 2 \delta) a(\delta)+\int_{0}^{\delta} \kappa_{(\infty)}(t ; 2 x) \alpha^{\prime}(x) d x .
$$

Nous récrivons (7.38):

$$
2 \pi^{2} t \kappa_{(\infty)}(t ; 2 x)=\frac{1}{2 \sqrt{2}}\left\{f_{7}(t ; x)+f_{9}(t ; x)\right\}+f_{11}(t ; x) .
$$

Nous voyons d'abord

$$
\int_{0}^{\delta} f_{11}(t ; x) a^{\prime}(x) d x=O(\sqrt{t}), \quad \text { grâce à }(7.39) \text {. }
$$

Ensuite,

$$
\frac{1}{2 \sqrt{2}} \int_{0}^{\delta} f_{7}(t ; x) a^{\prime}(x) d x=\frac{\pi}{2} \log \frac{1}{t} a(\delta)+\text { Cte. }+O(t),
$$

d'après le Lemme 7.4. Considérons troisièmement

$$
\int_{0}^{\delta} f_{9}(t ; x) a^{\prime}(x) d x=\int_{0}^{\left.t / 2 \xi_{1}\right)} f_{9}(t ; x) a^{\prime}(x) d x+\int_{t /\left(2 \xi_{1}\right)}^{\delta} f_{9}(t ; x) a^{\prime}(x) d x .
$$

D'après (7.35) du Lemme 7.6, nous avons

le premier terme à droite de $(7.44)=O(t \log (1 / t))$.

Et, par (7.36) du même Lemme,

le dernier terme à droite de $(7.44)=\int_{t /\left(2 \xi_{1}\right)}^{\delta} f_{9}(0 ; x) a^{\prime}(x) d x+R$, où $|R| \leqq$ Cte. $\int_{t /\left(2 \xi_{1}\right)}^{\delta}(t / x)^{3 / 2} \exp (-c x / t) d x=O(t)(c=$ Cte. $>0)$,

$$
\text { et } \int_{t /\left(2 \xi_{1}\right)}^{\delta} f_{9}(0 ; x) a^{\prime}(x) d x=\text { Cte. }+O(t \log (1 / t)) \text {, }
$$

parce que $f_{9}(0 ; x)=O(\log (1 / x))$ si $x>0$ est petit. Donc, en somme,

$$
\int_{0}^{\delta} f_{9}(t ; x) a^{\prime}(x) d x=\text { Cte. }+O(t \log (1 / t)) .
$$

Par (7.42), (7.43) et $\left(7.44^{\prime}\right)$, nous avons 


$$
\begin{aligned}
& 2 \pi^{2} t \int_{0}^{\delta} \kappa_{(\infty)}(t ; 2 x) a^{\prime}(x) d x=\frac{\pi}{2} a(\delta) \log (1 / t)+\text { Cte. }+O(\sqrt{t}) \\
& \quad=2 \pi^{2} t \kappa_{(\infty)}(t ; 2 \delta) a(\delta)+\text { Cte. }+O(\sqrt{t}),
\end{aligned}
$$

d'où le résultat.

C.Q.F.D.

CoRollaire 7.4. Soit $n=2$. Désignons par $A(\delta), B(\delta)$, et $C(\delta)$ les constantes $A, B$ et $C$ dans (7.7), (7.9) et (7.40) respectivement. Alors, nous avons

$$
\begin{aligned}
& A(\delta)=\frac{1}{4 \pi}\{\log (8 \delta)+\gamma\}, \\
& B(\delta)=\frac{1}{4 \pi}\{\log (2 / \delta)-\gamma\}, \\
& C(\delta)=\frac{1}{4 \pi} \int_{0}^{\delta} \frac{a(x)}{x} d x,
\end{aligned}
$$

où $\gamma=-\Gamma^{\prime}(1)$ est le nombre d'Euler.

PreUve. Comme $\psi_{3}(0)=A(\delta)+B(\delta)$, il suffit de savoir les valeurs $\psi_{3}(0)$ et $B(\delta)$. (7.47) est une conséquence de (7.41) qui implique $C(\delta)=$ $-2 \int_{0}^{\delta} B^{\prime}(2 x) a(x) d x$. D'abord, (7.4) et un calcul simple donnent

$$
\psi_{3}(0)=\frac{1}{\pi} \log 2 .
$$

D'autre part, (7.20), (7.25) (7.29), (7.31) (7.33) et (7.37) nous donnent

$$
\begin{aligned}
4 \pi B(\delta)= & \frac{1}{\sqrt{2 \pi}} f_{9}(0)+\left\{\frac{1}{\sqrt{2 \pi}} f_{7}(t)-\log \frac{1}{t}\right\}_{t=0} \\
= & \frac{1}{\sqrt{\pi}} \int_{\delta}^{\infty} e^{-x} \Psi(1 / 2,1 / 2 ; X) \frac{d X}{X}+\log 8 \\
& -\frac{2}{\sqrt{\pi}} \int_{0}^{\delta}{ }_{1} F_{1}(1 / 2 ; 3 / 2 ;-X) \frac{d X}{\sqrt{X}}
\end{aligned}
$$

Mais on sait que

$$
\begin{aligned}
& \int_{0}^{\infty} e^{-X} \Psi(1 / 2,1 / 2 ; X) \frac{d X}{X}-2 \int_{0}^{\delta}{ }_{1} F_{1}(1 / 2,3 / 2 ;-X) \frac{d X}{\sqrt{X}} \\
& \quad=\int_{0}^{\infty} e^{-x}\left(\log \frac{X}{\delta}\right) \frac{d X}{\sqrt{X}}=\Gamma^{\prime}(1 / 2)-\sqrt{\pi} \log \delta=-\sqrt{\pi}(\gamma+2 \log 2+\log \delta),
\end{aligned}
$$

d'où (7.46).

C.Q.F.D. 


\section{BIBLIOGRAPHIE}

[1] M. S. Baouendi Et C. Goulaouic, Régularité et théorie spectrale pour une classe d'opérateurs elliptiques dégénérés. Arch. Rat. Mec. Anal. 34 (1969), 361-378.

[2] S. D. Eidelman, Parabolic systems. (English Translation) North-Holland Publ. Comp., Wolters-Noordhoff Publ. Comp.

[3] A. ERdeliyi, W. Magnus, F. Oberhettinger et F. G. Tricomi, Higher transcendental functions, Vol. 1. McGraw-Hill.

[4] S. Minakshisundaram, et A. Pleijel, Some properties of the eigenfunctions of the Laplace operator on Riemannian manifold. Canad. J. Math. 1 (1949), 242-256.

[5] C. NoRdin, The asymptotic distribution of the eigenvalues of a degenerate elliptic operator. Univ. Lund, Lund Inst. Tech., 1971.

[6] L. Schwartz, Théorie des distributions. Hermann, Paris.

[7] N. Shimakura, Quelques exemples des $\zeta$-fonctions d'Epstein pour les opérateurs elliptiques dégénérés du second ordre. I et II. Proc. Japan Acad. Vol. 45, $N^{\circ} 10$ (1969) 866-871 et Vol. 46, $\mathrm{N}^{\circ} 10$ (1970) 1065-1069.

[8] N. Shimakura, Les fonctions de Green pour certains opérateurs paraboliques dégénérés dans le demi-espace. Proc. Japan Acad. Vol. 47, $\mathrm{N}^{\circ} 9$ (1971) 699-704.

Mathematical Institute

TÔHOKU UNIVERSITY

SENDAI, JAPAN 
